\title{
The fourth catalogue of Population I Wolf-Rayet stars in the Large Magellanic Cloud ${ }^{\star}$
}

\author{
J. Breysacher ${ }^{1}$, M. Azzopardi ${ }^{2}$, and G. Testor ${ }^{3}$ \\ 1 European Southern Observatory, Karl-Schwarzschild-Strasse 2, D-85748 Garching bei München, Germany \\ 2 Observatoire de Marseille, 2 place Le Verrier, F-13248 Marseille Cedex 04, France \\ 3 Observatoire de Paris, Section de Meudon, DAEC, F-92195 Meudon Cedex, France
}

Received December 14, 1998; accepted March 1, 1999

\begin{abstract}
The catalogue provides for each of the 134 W-R stars of Population I presently known in the Large Magellanic Cloud, accurate equatorial coordinates, photometric data, spectral classification, binary status, correlation with $\mathrm{OB}$ associations and HII regions. The miscellaneous designations of the stars are also listed. Although completeness is not pretended, results published during the last decade are highlighted in the notes given for each individual star. A uniform set of finding charts is presented.
\end{abstract}

Key words: Catalogs — galaxies: Magellanic Clouds galaxies: stellar content — stars: Wolf-Rayet

\section{Introduction}

The first systematic search for Wolf-Rayet $(\mathrm{W}-\mathrm{R})$ stars in the Large Magellanic Cloud (LMC) was carried out by Westerlund \& Rodgers (1959). Their observational material consisted of 40 blue-sensitive plates taken with an objective prism attached to the $50 \mathrm{~cm}$ Schmidt telescope, at the Uppsala Southern Station of the Mount Stromlo Observatory. The reciprocal dispersion of the spectra was $380 \AA \mathrm{mm}^{-1}$ at $\mathrm{H} \gamma$. This search yielded 50 objects of which 36 were in common with the Harvard spectral surveys (Cannon 1924, 1933; Payne 1930).

Later on, Westerlund \& Smith (1964) repeated the survey of the same field of about 100 square degrees and published a list of $58 \mathrm{~W}-\mathrm{R}$ stars. These were then considered to represent the quasi totality of the LMC W $-\mathrm{R}$ population. In this first catalogue the stars were roughly

Send offprint requests to: J. Breysacher

* Figures 2 to 12 only in the electronic version at http://edpsciences.com classified $\mathrm{C}$ (carbon-type), $\mathrm{N}$ (nitrogen-type) or $\mathrm{W}+\mathrm{O}$ (binaries); the more accurate spectral types previously assigned to 15 of them by Feast et al. (1960) being however given. The article included a study of the LMC W $-\mathrm{R}$ star surface distribution as well as a discussion of some fundamental physical parameters of the stars, such as luminosity, mass and age. For about $65 \%$ of these $\mathrm{W}-\mathrm{R}$ stars, spectral types were subsequently presented by Smith (1968b) who also derived mean absolute magnitudes and intrinsic colours for the stars in the various WN and WC sub-classes.

However, further discoveries indicated that the census of the LMC $\mathrm{W}-\mathrm{R}$ population was still not complete. In his catalogue of 1272 proven or probable LMC member stars, Sanduleak (1969) denoted for instance as "WR" 14 objects which were not listed by Westerlund \& Smith (1964).

The careful inspection of plates obtained with the ESO $40 \mathrm{~cm}$ Objective Prism Astrograph (GPO) - primarily secured for radial velocity measurements - allowed Fehrenbach et al. (1976) to recognize all the stars identified by Westerlund \& Smith, to confirm 12 of the 14 Sanduleak $\mathrm{W}-\mathrm{R}$ candidates and to discover 6 new $\mathrm{W}-\mathrm{R}$ stars. This second catalogue of LMC $\mathrm{W}-\mathrm{R}$ stars included 76 classified objects, 29 of which received a spectral type for the first time. A discrimination between the WN3, WN4 and WN5 sub-classes being nevertheless impossible from the objective prism plates, in the nitrogen sequence, most of the stars were only classified WN3-5 by Fehrenbach et al. (1976).

Walborn (1977) added a new member to the LMC $\mathrm{W}-\mathrm{R}$ population by finding that the star Sk- $67^{\circ} 18$ - classified Of by Sanduleak (1969) and O9f by Ardeberg et al. (1972) - has a companion of type WN5-6; and Melnick (1978) increased to 83 the number of known $\mathrm{W}-\mathrm{R}$ stars in the LMC with the detection of 6 additional $\mathrm{W}-\mathrm{R}$ stars near the core of the 30 Doradus nebula. 
Finally, Azzopardi \& Breysacher $(1979,1980)$, as a result of a new systematic search for $\mathrm{W}-\mathrm{R}$ stars in the LMC with the ESO $40 \mathrm{~cm}$ GPO, using an interference filter centered at $\lambda 4650$, were able to identify 17 new such objects in this galaxy. The LMC area covered by this survey ( 23 partially overlapping fields, each 85 arcmin in diameter) is shown in Fig. 1 of the paper by Azzopardi \& Breysacher (1980). The third catalogue of LMC W - R stars was published by Breysacher in 1981. It provided accurate spectral types for all the $100 \mathrm{~W}-\mathrm{R}$ stars then known in this galaxy. For $92 \%$ of the objects the classification was based on slit spectrograms obtained at the $3.6 \mathrm{~m}$ and $1.5 \mathrm{~m}$ ESO telescopes by the author.

Since the publication of the Breysacher's catalogue, the number of $\mathrm{W}-\mathrm{R}$ stars identified in the $\mathrm{LMC}$ has again increased. Additional $\mathrm{W}-\mathrm{R}$ stars have been discovered in the field either as the result of new systematic searches of deep objective-prism plates covering a larger area (Morgan \& Good 1985, 1990), or by serendipity (Cowley et al. 1984; Azzopardi \& Breysacher 1985; Morgan \& Good 1987; Morgan 1999). Others were detected or resolved in compact clusters/HII regions (Weigelt \& Baier 1985; Moffat et al. 1987; Heydari-Malayeri et al. 1990; Testor \& Schild 1990; Schild \& Testor 1992; Heydari-Malayeri et al. 1993; Walborn et al. 1995). The recognition of the genuine or borderline $\mathrm{W}-\mathrm{R}$ nature of some already known stars (Conti \& Garmany 1983; Bohannan \& Walborn 1989; Walborn \& Blades 1997) also contributed to the growth of the LMC W $-\mathrm{R}$ population.

The fact that for these objects, neither a complete set of finding charts nor equatorial coordinates with the accuracy required for observations with modern ground-based telescopes or spacecraft exist, has been an incentive for the preparation of this updated fourth catalogue of $\mathrm{W}-\mathrm{R}$ stars in the LMC, which now contains 134 objects.

\section{Astrometric measurements}

For $87 \%$ of the stars listed in the catalogue, the right ascension and declination were determined from 15 direct B plates (IIIa-J emulsion; scale: $50 \mathrm{arcsec} / \mathrm{mm}$ ) obtained with the ESO $40 \mathrm{~cm}$ GPO, at La Silla. The measurements were performed with the OPTRONICS machine at ESO-Garching. The data were reduced using the POS1 astrometric programme written by West (1981), with the implementations added by O. Hainaut. An average of 30 reference stars per plate, selected from the PPM catalogue (Bastian et al. 1991), was used. Morgan (1999) provided to us the 2000.0 coordinates of the new $\mathrm{W}-\mathrm{R}$ star (MG 9) he recently found in the LMC.

For $9 \%$ of the stars, located in crowded regions and therefore difficult to measure on the photographic plates, the positions were determined on CCD images (HeII 4686 interference filter; scale: $0.26 \mathrm{arcsec} / \mathrm{pixel}$ ) secured with the direct camera attached to the $2.2 \mathrm{~m}$ telescope, at La Silla. The equatorial coordinates of these $\mathrm{W}-\mathrm{R}$ stars were calculated by linear interpolation using as secondary standards nearby stars measured on the astrographic plates. The central stellar positions were established using a two-dimensional Gaussian fit. For the remaining $4 \%$ of the stars - six members of the $\mathrm{R} 136$ cluster at the heart of the 30 Doradus nebula - we adopted the coordinates published by Malumuth \& Heap (1994).

For the stars appearing on different plates or CCD frames, the astrometric measurements were repeated. This resulted in $30 \%$ and $10 \%$ of the stars having their coordinates determined independently two times and three times respectively. An estimation of the internal error is therefore possible. The obtained accuracy is $\pm 0.045 \mathrm{~s}$ in right ascension, and \pm 0.20 arcsec in declination. For those stars in 30 Doradus where the comparison is possible, our coordinates are in excellent agreement with the ones given by Parker (1993) and Malumuth \& Heap (1994).

\section{Spectral classification}

It is perhaps worthwhile to recall that spectral types have been, and are still, assigned to $\mathrm{W}-\mathrm{R}$ stars exclusively on the basis of the morphological properties of their emission line spectra, according to the classification scheme originally introduced by Beals (1938). Following this scheme, the $\mathrm{W}-\mathrm{R}$ stars are arranged into two parallel sequences: one in which the helium and nitrogen lines dominate the optical spectrum, the WN sequence; and another one in which, besides the helium lines, strong carbon and oxygen emissions are observed, the WC sequence. In the scheme adopted by the IAU Commission 29 (Beals 1938), ranging from the highest to the lowest excitation, only the subtypes WN5 to WN8 and WC6 to WC8 were considered. Subsequent systematic investigations of the spectra of $\mathrm{W}-\mathrm{R}$ stars in the Galaxy and the Magellanic Clouds (Hiltner \& Schild 1966; Smith 1968a; Walborn 1974; Breysacher 1981; van der Hucht et al. 1981; Massey \& Conti 1983b; Torres et al. 1986; van der Hucht et al. 1988; Conti \& Massey 1989; Pakull 1991; Smith et al. 1994; Smith et al. 1996) led to a refinement of the W - R classification and to the inclusion of additional subtypes in both the WN and WC sequences which now extend from WN1 to WN11 and WC4 to WC11 respectively.

In order to distinguish, in the WC sequence, those of the $\mathrm{W}-\mathrm{R}$ stars which exhibit unusually strong oxygen lines, Barlow \& Hummer (1982) have proposed to have a third sequence called WO. According to these authors, WO stars can be divided into four subtypes, WO1 to WO4, mainly defined by the relative strengths of the OIV $\lambda 3400$, OV $\lambda 5590$ and OVI $\lambda \lambda 3811,3834$ lines, the lowest excitation lines of oxygen being observed in the spectra of WO4 $\equiv$ WC4 stars. Rustamov \& Cherepashchuk (1987) introduced later on the subtype WO5. 
In the LMC, there are stars for which neither an Of- nor a WN-type unambiguously account for the observed spectral features. A fourth generic category designated as "Of/WN" allows to separate these objects from the classical WN stars. The spectral type Ofpe/WN9, interpreted as an extension to later types of the WN sequence, was originally assigned by Bohannan \& Walborn (1989) to ten LMC stars. Most of them have now been reclassified WN10 or WN11 by Crowther \& Smith (1997). These stars are believed to represent the minimum or quiescent state of Luminous Blue Variables (Smith et al. 1998), hereafter LBVs. A few other LMC stars classified O3If*/WN6-A (Walborn 1982a, 1986; Walborn \& Blades 1997; Massey \& Hunter 1998) also enter the Of/WN category.

With the advent of linear area detectors the quantification of the $\mathrm{W}-\mathrm{R}$ classification system has been greatly facilited, allowing the determination of objective spectral types for WN2-8 stars (Smith et al. 1996), WN9-11 stars (Smith et al. 1994), WC4-9 stars (Smith et al. 1990b), WO1-5 stars (Kingsburgh et al. 1995), WC4-11 and WO1-4 (Crowther et al. 1998). The spectral types adopted in the present catalogue originate primarily from such quantitative studies; the most recent works being given preference. The three-dimensional scheme for WN stars introduced by Smith et al. (1996), and currently employed by others (e.g. Crowther \& Smith 1997; Morgan 1999), is largely used. For those stars which are not yet classified according to these quantitative schemes, we have selected in the literature the best possible determined spectral types, or at least, estimated as such. Whenever available, other classification work references are provided in the catalogue.

In the classification of $\mathrm{W}-\mathrm{R}$ stars, the question of companions still remains a delicate issue. Although the only secure method to establish the binary nature of a $\mathrm{W}-\mathrm{R}$ star is to search for radial velocity variations and/or eclipses, two other criteria, namely "small ratio of emission-line to continuum intensities" and "presence of absorption lines" are sometimes used too. Several detailed investigations of galactic W-R stars (see Fitzpatrick 1982, and references quoted therein) having shown that these spectral characteristics are also exhibited by single $\mathrm{W}-\mathrm{R}$ stars, it is a bit hazardous to assume that $\mathrm{W}-\mathrm{R}$ stars of similar types necessarily have similar spectral appearance in the absence of a companion. Binarity deduced from the use of these criteria only is therefore doubtful. Although the LMC is a system seen nearly "face on", and not much extended in depth (de Vaucouleurs \& Freeman 1972), it is risky as well, to conclude that a $\mathrm{W}-\mathrm{R}$ star is a binary exclusively on the basis of its high luminosity, in view of the absolute-magnitude distribution obtained for the single WN- and WC-type stars in the LMC (Breysacher 1986).

In Fig. 1, we present spectra of LMC $\mathrm{W}-\mathrm{R}$ stars representative of the WNE, WNL (cf. Vanbeveren \&
Conti 1980) and WCE (cf. Torres et al. 1986) subclasses. The spectrograms were obtained, in 1980, with the Boller \& Chivens spectrograph equipped with an Image Dissector Scanner, at the Cassegrain focus of the ESO $3.6 \mathrm{~m}$ telescope, at La Silla. The reciprocal dispersion used was $172 \AA \mathrm{mm}^{-1}$. The main emission lines present in the observed spectral range $(4000-7500 \AA)$ are identified.

\section{The catalogue}

The fourth catalogue of LMC $\mathrm{W}-\mathrm{R}$ stars is presented in Table 2 and contains 134 objects; each of them has been, at least once, assigned a $\mathrm{W}-\mathrm{R}$ type. The miscellaneous designations of the stars from discovery papers are given in Table 3. Finding charts for all the stars appearing in the catalogue are provided in Figs. 2-12.

\section{Table 2: Format of stellar data}

Column 1: Running catalogue number.

Column 2: The designation "Brey" refers to the third catalogue of LMC W - R stars published by Breysacher (1981). The letters "a", "b" and "c" denote the stars subsequently included in this catalogue.

Column 3: Equatorial coordinates for J2000.0. The accuracy is $\pm 0.045 \mathrm{~s}$ in right ascension, and \pm 0.20 arcsec in declination.

Column 4: $v$ magnitude in narrow-band photometry in Smith's (1968b) system. Boldfaced values correspond to the original measurements by Smith (1968b), the other values are Johnson $V$ magnitudes corrected for emission line contamination using the correction factors determined by Breysacher (1986). When available, the synthetic $v$ magnitude derived by Torres-Dodgen \& Massey (1988) is given in parentheses. Sources for the $V$ magnitudes are as follows:

a: Rousseau et al. (1978)

b: Azzopardi \& Breysacher $(1979,1980,1985)$

c: Feitzinger \& Isserstedt (1983)

d: Morgan \& Good $(1985,1987,1990)$

e: Bohannan \& Walborn (1989)

f: Lee (1990)

g: Schild \& Testor (1992)

h: Parker (1993)

i: Testor et al. (1993)

j: Malumuth \& Heap (1994)

k: Spoon et al. (1994)

l: Schertl et al. (1995)

m: Walborn \& Blades (1997)

n: Morgan (1999) 
o: present work.

Column 5: $b-v$ colour in narrow-band photometry in Smith's (1968b) system. Boldfaced values correspond to the original measurements by Smith $(1968 \mathrm{~b})$, the other values are the synthetic colours derived by Torres-Dodgen \& Massey (1988).

Column 6: Spectral classification. The reference number given in parentheses refers to the source (cf. Col. 7).

Column 7: Bibliographical references for the spectral classification of the star. The sources, in chronological order, are codified as follows:

60: Feast et al. (1960)

68: Smith (1968b)

76: Fehrenbach et al. (1976)

77: Walborn (1977)

78: Melnick (1978)

81: Breysacher (1981)

82a: Conti (1982)

82b: Phillips (1982)

82c: Walborn (1982a)

83a: Massey \& Conti (1983a)

83b: Conti et al. (1983)

83c: Conti \& Garmany (1983)

83d: Mathewson et al. (1983)

84: Cowley et al. (1984)

85a: Azzopardi \& Breysacher (1985)

85b: Morgan \& Good (1985)

85c: Melnick (1985)

86a: Walborn (1986)

86b: Moffat \& Seggewiss (1986)

87a: Moffat et al. (1987)

87b: Morgan \& Good (1987)

88a: Breysacher (1988)

88b: Torres-Dodgen \& Massey (1988)

89a: Conti \& Massey (1989)

89b: Bohannan \& Walborn (1989)

89c: Moffat (1989)

90a: Moffat et al. (1990)

90b: Smith et al. (1990a)

90c: Morgan \& Good (1990)

90d: Heydari-Malayeri et al. (1990)

90e: Testor \& Schild (1990)

91a: Niemela (1991)

91b: Pakull (1991)

92a: Schild \& Testor (1992)

92b: Heydari-Malayeri \& Melnick (1992)

93a: Heydari-Malayeri et al. (1993)

93b: Testor et al. (1993)

94 : Heap et al. (1994)

95a: Bartzakos et al. (1995)

95b: Niemela (1995) 95c: Niemela et al. (1995)

95d: Crowther et al. (1995)

95e: Schertl et al. (1995)

96a: Brandl et al. (1996)

96b: Smith et al. (1996)

97a: Crowther \& Smith (1997)

97b: Walborn \& Blades (1997)

98a: Massey \& Hunter (1998)

98b: Crowther et al. (1998)

98c: Crowther \& Dessart (1998)

98d: Testor \& Niemela (1998)

98e: Morgan (1999)

98f: Bartzakos (1998).

Column 8: Binarity of the star. SB1 and SB2 designate, as usual, the single-lined and double-lined spectroscopic binaries respectively. The number in parentheses refers to the source (cf. Col. 7). A question mark (?) indicates that binarity remains to be confirmed.

Column 9: OB association, as defined by Lucke \& Hodge (1970), to which the $\mathrm{W}-\mathrm{R}$ star very likely belongs.

Column 10: Emission nebula where the $\mathrm{W}-\mathrm{R}$ star lies. Both the Henize (1956) number - when available - and the DEM (Davies et al. 1976) number are given.

Column 11: Notes on individual stars are indicated with an asterisk. The presence of two asterisks identifies the few objects for which the classification varies from $\mathrm{W}-\mathrm{R}$ to non $\mathrm{W}-\mathrm{R}$ in the literature, or is controversial.

\section{Table 3: Format of designations}

To comply with the IAU nomenclature recommendations, the first line in Table 3 refers to the designations adopted in the Dictionary of Nomenclature of Celestial Objects. Only the acronyms in Cols. 12, 16 and 20, proposed here for the first time, are not yet "official", and therefore appear between parentheses. On the second line, when different, the more traditionally used acronyms are given for convenience. The designations, in chronological order from Col. 2, are listed in Table 1.

\section{Finding charts}

Our goal has been to present finding charts (Figs. 2-12) as uniform as possible for the greatest number of objects. Except for 30 Doradus (Fig. 12) and the enlargements of the compact clusters, the charts have a $3 \times 3$ arcmin field. They are all oriented with north at the top and east to the left.

The Digital Sky Survey (DSS) was used for $70 \%$ of the stars. The charts corresponding to the MG stars (Morgan \& Good 1985; Morgan 1999); BAT99-20, 
Table 1.

\begin{tabular}{|c|c|c|}
\hline Column & Designation & References \\
\hline 1 & BAT99 & : Running catalogue number (IAU registered) \\
\hline 2 & $\mathrm{HD} / \mathrm{HDE}$ & : Henry Draper Catalogue and its Extension \\
\hline 3 & LHA $120-\mathrm{S}=\mathrm{S}$ & : Henize (1956) \\
\hline 4 & $\mathrm{RMC}=\mathrm{R}$ & $\begin{array}{l}\text { : Feast et al. (1960). Subdivisions in R } 136 \text { and R } 140 \\
\text { are from Weigelt \& Baier (1985) and Moffat et al. (1987) respectively. }\end{array}$ \\
\hline 5 & $\mathrm{~W} 61=\mathrm{W}$ & : Westerlund (1961), (table number-star number) \\
\hline 6 & {$[\mathrm{~L} 63]=\mathrm{L}$} & : Lindsay (1963) \\
\hline 7 & $\mathrm{AL}$ & : Andrews \& Lindsay (1964) \\
\hline 8 & WS & : Westerlund \& Smith (1964) \\
\hline 9 & $\mathrm{Sk}$ & : Sanduleak (1969) \\
\hline 10 & {$[\mathrm{BE} 74]=\mathrm{BE}$} & : Bohannan \& Epps (1974) \\
\hline 11 & FD & : Fehrenbach et al. (1976) \\
\hline 12 & $(\mathrm{Mk} 78)=\mathrm{Mk}$ & : Melnick (1978) \\
\hline 13 & $\mathrm{LMC} \mathrm{AB}=\mathrm{AB}$ & : Azzopardi \& Breysacher $(1979,1980,1985)$ \\
\hline 14 & Brey & : Breysacher (1981) \\
\hline 15 & $\mathrm{MGWR}=\mathrm{MG}$ & : Morgan \& Good (1985), Morgan (1999) \\
\hline 16 & $(\mathrm{Mk} 85)=\mathrm{Mk}$ & : Melnick (1985) \\
\hline 17 & TSWR & : Testor \& Schild (1990), Schild \& Testor (1992) \\
\hline 18 & {$[\mathrm{CHH} 92]=\mathrm{CH}$ or $\mathrm{WF}$} & : Campbell et al. (1992) \\
\hline 19 & {$[\mathrm{P} 93]=\mathrm{P}$} & : Parker (1993) \\
\hline 20 & $(\mathrm{MaH})$ & : Malumuth \& Heap (1994) \\
\hline 21 & [HSH95] & : Hunter et al. (1995) \\
\hline
\end{tabular}

23, 25, 81, 121, and to BAT99-129, were made from SuperCOSMOS scans of UKST plates kindly made available to us by D.H. Morgan.

For stars located in crowded regions we had to resort to other sources. CCD frames obtained at La Silla were used for BAT99-31, 67, 68, 69, 70, 71, 72, 73, 74, 76, 77, 78, 79, 80, 120, 125, 126, 127 (3.6 m telescope + EFOSC1, $U$ filter), BAT99-42 (3.6 m telescope + EFOSC1, HeII filter), BAT99-88, 89, 91 (3.6 m telescope + EFOSC1, $V$ filter); and for BAT99-28, 83, 124 (2.2 m telescope + EFOSC2, HeII filter). The 30 Doradus image was provided by G. Meylan, it corresponds to a $3 \mathrm{~s} \mathrm{~V}$ CCD exposure obtained in 0.5 arcsec seeing, with the $2.2 \mathrm{~m}$ telescope at La Silla (Meylan 1993).

For BAT99-10, 68, 69, 77, 78, 80, 91, 106, 108, 109, $110,111,112$, the high-resolution images were retrieved from the HST archive.

\section{Notes on individual stars}

These notes provide for 114 stars of the catalogue a sort of "identity card", i.e. a short summary of the most important results published over the past years. Our survey of the literature covers essentially the last decade. Although we have attempted to be as complete as possible in this compilation, it cannot be excluded that a few papers escaped our attention.
BAT99-1 (Brey 1): Due to the weakness of the NIV $\lambda 3480$ feature, Conti \& Massey (1989) suggested that the spectral type WN2.5 or even WN2 could possibly be assigned to this star.

From an analysis of the helium spectrum, Koesterke et al. (1991) have derived the following set of values for the "core radius" $R_{*}$, the related effective temperature $T_{*}$, the luminosity $L$ and the mass-loss rate $\dot{M}$ of the star:

$R_{*}=1.9 R_{\odot} ; T_{*}=73000 \mathrm{~K} ; \log \left(L / L_{\odot}\right)=5.0 ;$ $\log \left(\dot{M} /\left(M_{\odot} \mathrm{yr}^{-1}\right)\right)=-4.8$.

$R_{*}=1.3 R_{\odot} ; T_{*}=90000 \mathrm{~K}$ (adopted); $\log \left(L / L_{\odot}\right)=5.0 ;$ $\log \left(\dot{M} /\left(M_{\odot} \mathrm{yr}^{-1}\right)\right)=-4.6$.

The uncertainty results from the weakness of the HeI $\lambda 5876$ emission line identified as a slightly increased continuum of $9 \AA$ equivalent width.

According to Morris et al. (1993), the continuum energy distribution of the star can be fitted by a power law of the form $F_{\lambda} \sim \lambda^{-\alpha}$, where $\alpha=2.04$, over the range $1500-9000 \AA$. A spectrum of the star in the range $4500-6700 \AA$ is shown in Fig. 1 of the paper by Morgan, Watson \& Parker (1992).

BAT99-2 (Brey 2): This star lies in the HeIII region discovered within the N 79 nebula by Pakull (1991) who assigned to the star the earliest WN type: WN1. An image of the region is given by Melnick \& Heydari-Malayeri (1991). 
According to Dopita et al. (1994), the ring nebula associated with the $\mathrm{W}-\mathrm{R}$ star is $23^{\prime \prime}$ in diameter, has similar morphology to NGC 6888 , and is brightest to the northwest. The HII region N 79 has a fine filamentary loop about $3^{\prime}$ in diameter.

Garnett \& Chu (1994) reported that the W - R ejecta shell shows $\mathrm{He}$ and $\mathrm{N}$ abundances identical to the LMC interstellar values.

BAT99-3 (Brey 3): According to Morris et al. (1993), the continuum energy distribution of the star can be fitted by a power law of the form $F_{\lambda} \sim \lambda^{-\alpha}$, where $\alpha=2.84$, over the range $1500-9000 \AA$. This star is a borderline WN3 (Smith et al. 1996).

BAT99-4 (Brey 3a): According to Heydari-Malayeri et al. (1990), the nebula N 82 presents chemical abundances anomalies: nitrogen is enriched with respect to the average LMC value by a factor of 5 , whereas oxygen is depleted by a factor 2.5. This very compact HII region seems to have been ejected by the central WC9-type $\mathrm{W}-\mathrm{R}$ star which is likely to be a member of a binary system, with an O-type companion. The ionizing source for $\mathrm{N} 82$ is estimated to have an absolute magnitude $M_{v}=-4.5$ and an effective temperature $T_{\text {eff }}=36000 \mathrm{~K}$.

Moffat (1991) argued that the emission-line spectrum of $\mathrm{N} 82$ does not contain a WC9 spectrum, a WNL or Of spectrum appears more likely.

On the basis of new observations, Heydari-Malayeri \& Melnick (1992) indicated that the central star (or stars) of $\mathrm{N} 82$ does not seem to be a canonical WC9 subclass.

The object is designated as Brey 3a by Lortet (1991).

BAT99-5 (Brey 4): No optical nitrogen ion lines being visible, Conti \& Massey (1989) suggested that the spectral type WN1 could possibly be assigned to this star.

From an analysis of the helium spectrum, Koesterke et al. (1991) have derived the following set of values for the "core radius" $R_{*}$, the related effective temperature $T_{*}$, the luminosity $L$ and the mass-loss rate $\dot{M}$ of the star:

$R_{*}=1.8 R_{\odot} ; T_{*}=47000 \mathrm{~K} ; \log \left(L / L_{\odot}\right)=4.2 ;$ $\log \left(\dot{M} /\left(M_{\odot} \mathrm{yr}^{-1}\right)\right)=-5.1$.

$R_{*}=1.2 R_{\odot} ; T_{*}=90000 \mathrm{~K}$ (adopted); $\log \left(L / L_{\odot}\right)=4.9$; $\log \left(\dot{M} /\left(M_{\odot} \mathrm{yr}^{-1}\right)\right)=-4.8$.

The uncertainty results from the weakness of the HeI $\lambda 5876$ emission line $\left(W_{\lambda}=8 \AA\right)$.

A spectrum of the star in the range $4200-5300 \AA$ is shown in Fig. 1 of the paper by Morgan et al. (1992).

BAT99-6 (Brey 5): The star was classified O9f by Ardeberg et al. (1972). According to Walborn (1977), the broad HeII $\lambda 4686$ and narrow NIV $\lambda 4058$ emission present, indicate a WN component.

Dopita et al. (1994) noted that the star is embedded in the filamentary superbubble $\mathrm{N} 9$; there is a filament of high excitation running in a north-south direction which clearly passes close to the $\mathrm{W}-\mathrm{R}$ star. No classical ring nebula is seen.

According to Niemela et al. (1995), the object appears to be a high-mass $\left(\sim 80 M_{\odot}\right)$ multiple system containing an eclipsing binary ( $P \simeq 2$ days) which primary component is not of spectral type WN but probably of type O3f*.

BAT99-7 (Brey 6): Breysacher (1981) classified this star as peculiar because of the unusually broad emission lines. Koesterke et al. (1991) derived the following values for the "core radius" $R_{*}$, the related effective temperature $T_{*}$, the luminosity $L$ and the mass-loss rate $\dot{M}$ of the star:

$R_{*}=2.5 R_{\odot} ; T_{*}=90000 \mathrm{~K} ; \log \left(L / L_{\odot}\right)=5.6$; $\log \left(\dot{M} /\left(M_{\odot} \mathrm{yr}^{-1}\right)\right)=-3.8$.

These authors point out that the spectrum exhibits a very strong HeII emission at $5412 \AA$ which has a larger equivalent width than the maximum reached in their standard grid of models. Therefore, they had to perform special calculations with $16 \mathrm{HeII}$ levels for the analysis of this star.

According to Morris et al. (1993), the continuum energy distribution of the star can be fitted by a power law of the form $F_{\lambda} \sim \lambda^{-\alpha}$, where $\alpha=2.44$, over the range $1500-9000 \AA$.

BAT99-8 (Brey 8): Fehrenbach et al. (1976) noticed that star BE-159 is identified with WS 4 by Bohannan \& Epps (1974) while on their finding chart the corresponding star is not WS 4.

Dopita et al. (1994) reported the existence of a large, faint, previously unrecognized ring nebula visible in [OIII], which is seen most clearly to the north and to the southeast of the star. This ring is estimated to be $150^{\prime \prime} \times 230^{\prime \prime}$ in diameter.

According to Morris et al. (1993), the continuum energy distribution of the star can be fitted by a power law of the form $F_{\lambda} \sim \lambda^{-\alpha}$, where $\alpha=2.98$, over the range $1500-9000 \AA$.

BAT99-9 (Brey 7): Melnick \& Heydari-Malayeri (1991) reported that an HeII image suggests the presence of a faint HeIII region near the star.

According to Morris et al. (1993), the continuum energy distribution of the star can be fitted by a power law of the form $F_{\lambda} \sim \lambda^{-\alpha}$, where $\alpha=2.80$, over the range $1500-9000 \AA$.

A spectrum of this star is shown in Fig. 1c.

BAT99-10 (Brey 9): The multiple nature of this object was soon recognized (cf. Westerlund \& Smith 1964). The combined spectral type O9.5II:+WC5 assigned by Walborn (1977) could be clarified thanks to the speckle masking observations by Schertl et al. (1995). The core 
of the OB association LH 9 was resolved into 25 stellar components in a field of view of $6.4^{\prime \prime} \times 6.4^{\prime \prime}$, and star No. 2 was identified without ambiguity with the $\mathrm{W}-\mathrm{R}$ component (see Fig. 2). Another study of this cluster = HD32228 has been presented by Bauer et al. (1996).

BAT99-11 (Brey 10): Fehrenbach et al. (1976) noticed that star BE-171 is identified with WS 6 by Bohannan \& Epps (1974) while on their finding chart the corresponding star is not WS 6.

The star is in a superb ring nebula $\left(180^{\prime \prime} \times 112^{\prime \prime}\right.$ in diameter) discovered by $\mathrm{Chu}(1981)$; it has a measured expansion velocity of $45 \mathrm{~km} \mathrm{~s}^{-1}$ (Chu 1983).

According to Smith (1991), this star is twice as bright, in line and continuum, as any other WC4 star in the LMC.

BAT99-12 (Brey 10a): Star to which it is difficult to assign a unique spectral type. The notation $f^{*}$ is used by Walborn (1982a) to indicate that the NIV $\lambda 4058$ emission is stronger than NIII $\lambda 4634-4641$.

Niemela et al. (1991) reported that from their systematic search for high excitation nebulae around $\mathrm{W}-\mathrm{R}$ stars in the Magellanic Clouds, no evidence for nebular HeII $\lambda 4686$ emission in the vicinity of the star could be found.

BAT99-13: Crowther et al. (1995) derived the following values for the "core radius" $R_{*}$, the related effective temperature $T_{*}$, the luminosity $L$ and the mass-loss rate $\dot{M}$ of the star:

$R_{*}=20.5 R_{\odot} ; T_{*}=29700 \mathrm{~K} ; \log \left(L / L_{\odot}\right)=5.47$; $\log \left(\dot{M} /\left(M_{\odot} \mathrm{yr}^{-1}\right)\right)=-4.47$.

Pasquali et al. (1997) obtained for these parameters:

$R_{*}=19 R_{\odot} ; T_{*}=33000 \mathrm{~K} ; \log \left(L / L_{\odot}\right)=5.6$; $\log \left(\dot{M} /\left(M_{\odot} \mathrm{yr}^{-1}\right)\right)=-4.6$.

According to Morris et al. (1993), the continuum energy distribution of the star can be fitted by a power law of the form $F_{\lambda} \sim \lambda^{-\alpha}$, where $\alpha=2.09$, over the range $1500-9000 \AA$.

A spectrum of the star in the range $4000-4700 \AA$ is shown in Fig. 5 of the paper by Bohannan \& Walborn (1989).

BAT99-15 (Brey 12): Koesterke et al. (1991) derived the following values for the "core radius" $R_{*}$, the related effective temperature $T_{*}$, the luminosity $L$ and the mass-loss rate $\dot{M}$ of the star:

$R_{*}=2.3 R_{\odot} ; T_{*}=65000 \mathrm{~K} ; \log \left(L / L_{\odot}\right)=4.9 ;$ $\log \left(\dot{M} /\left(M_{\odot} \mathrm{yr}^{-1}\right)\right)=-4.3$.

According to Morris et al. (1993), the continuum energy distribution of the star can be fitted by a power law of the form $F_{\lambda} \sim \lambda^{-\alpha}$, where $\alpha=2.70$, over the range $1500-9000 \AA$.

Dopita et al. (1994) reported the discovery of the true ring nebula associated to this star, much a more compact filamentary shell, $103^{\prime \prime} \times 130^{\prime \prime}$ in diameter, than the $8^{\prime}$ across ring nebula claimed by Chu \& Lasker (1980).
BAT99-16 (Brey 13): Koesterke et al. (1991) derived the following values for the "core radius" $R_{*}$, the related effective temperature $T_{*}$, the luminosity $L$ and the mass-loss rate $\dot{M}$ of the star:

$R_{*}=15 R_{\odot} ; T_{*}=33000 \mathrm{~K} ; \log \left(L / L_{\odot}\right)=5.3$; $\log \left(\dot{M} /\left(M_{\odot} \mathrm{yr}^{-1}\right)\right)=-4.4$.

Crowther \& Smith (1997) obtained for these parameters: $R_{*}=16.7 R_{\odot} ; T_{*}=34800 \mathrm{~K} ; \log \left(L / L_{\odot}\right)=5.57$; $\log \left(\dot{M} /\left(M_{\odot} \mathrm{yr}^{-1}\right)\right)=-4.14$.

According to Morris et al. (1993), the continuum energy distribution of the star can be fitted by a power law of the form $F_{\lambda} \sim \lambda^{-\alpha}$, where $\alpha=2.81$, over the range $1500-9000 \AA$.

Dopita et al. (1994) reported the discovery of a fine ring nebula, only $30^{\prime \prime} \times 40^{\prime \prime}$ in diameter, probably representing stellar ejecta.

Garnett \& Chu (1994) derived $\mathrm{He} / \mathrm{H}$ and $\mathrm{N} / \mathrm{H}$ in the $\mathrm{W}-\mathrm{R}$ ejecta shell approximately a factor of two higher than the LMC HII region values, and N/O more than a factor of 10 higher than the characteristic LMC value.

BAT99-17 (Brey 14): According to Morris et al. (1993), the continuum energy distribution of the star can be fitted by a power law of the form $F_{\lambda} \sim \lambda^{-\alpha}$, where $\alpha=3.25$, over the range $1500-9000 \AA$.

BAT99-18 (Brey 15): According to Morris et al. (1993), the continuum energy distribution of the star can be fitted by a power law of the form $F_{\lambda} \sim \lambda^{-\alpha}$, where $\alpha=3.17$, over the range $1500-9000 \AA$.

BAT99-19 (Brey 16): Breysacher (1981) classified the star as peculiar because of the unusually broad emission lines.

Koesterke et al. (1991) derived the following values for the "core radius" $R_{*}$, the related effective temperature $T_{*}$, the luminosity $L$ and the mass-loss rate $\dot{M}$ of the star:

$R_{*}=6.2 R_{\odot} ; T_{*}=50000 \mathrm{~K} ; \log \left(L / L_{\odot}\right)=5.3 ;$ $\log \left(\dot{M} /\left(M_{\odot} \mathrm{yr}^{-1}\right)\right)=-4.1$.

According to Morris et al. (1993), the continuum energy distribution of the star can be fitted by a power law of the form $F_{\lambda} \sim \lambda^{-\alpha}$, where $\alpha=2.19$, over the range $1500-9000 \AA$.

Niemela et al. (1991) reported that from their systematic search for high excitation nebulae around $\mathrm{W}-\mathrm{R}$ stars in the Magellanic Clouds, no evidence for nebular HeII $\lambda 4686$ emission in the vicinity of the star could be found. According to Dopita et al. (1994), a faint filamentary nebula, $52^{\prime \prime} \times 64^{\prime \prime}$ in diameter, is associated with the star and appears only in [OIII].

BAT99-20 (Brey 16a): Dopita et al. (1994) noted that the star is surrounded by a small and bright ring nebula, $18^{\prime \prime}$ in diameter, seen best in [OIII].

According to Bartzakos (1998), the absorption-line 
spectrum might arise in a visual OB companion, therefore the star is considered as "almost certainly single".

BAT99-21 (Brey 17): According to Dopita et al. (1994), there is a faint nebula, $56^{\prime \prime}$ in diameter, associated with the star.

BAT99-22 (Brey 18): According to Allen \& Glass (1976), a late-type supergiant component provides the near-infrared flux. Cowley \& Hutchings (1978) confirmed the presence of said component spectroscopically and classified it M2Ia. Stahl et al. (1984) found that the star is related to the $\mathrm{S}$ Dor variables. According to Crowther et al. (1995), the star is probably a dormant LBV, or at a stage immediately after this phase.

Schmutz et al. (1991) derived the following values for the "core radius" $R_{*}$, the related effective temperature $T_{*}$, the luminosity $L$ and the mass-loss rate $\dot{M}$ of the star:

$R_{*}=30 R_{\odot} ; T_{*}=28500 \mathrm{~K} ; \log \left(L / L_{\odot}\right)=5.7 ;$ $\log \left(\dot{M} /\left(M_{\odot} \mathrm{yr}^{-1}\right)\right)=-4.6$.

Crowther et al. (1995) obtained very similar stellar parameters:

$R_{*}=33.8 R_{\odot} ; T_{*}=28500 \mathrm{~K} ; \log \left(L / L_{\odot}\right)=5.83$; $\log \left(\dot{M} /\left(M_{\odot} \mathrm{yr}^{-1}\right)\right)=-4.40$.

Pasquali et al. (1997) obtained for these parameters:

$R_{*}=25 R_{\odot} ; T_{*}=35200 \mathrm{~K} ; \log \left(L / L_{\odot}\right)=5.9$; $\log \left(\dot{M} /\left(M_{\odot} \mathrm{yr}^{-1}\right)\right)=-4.5$.

Dopita et al. (1994) pointed out that the arcs of nebulosity seen in $\mathrm{H} \alpha$ are not visible in [OIII]. Nota et al. (1996) reported the presence of strong nebular emission lines in the spectrum of BAT99-22 pointing out, however, that on the basis of the spectroscopic data alone no conclusion can be made about the physical association of the detected nebula with the star.

A spectrum of the star in the range $3900-4700 \AA$ is shown in Fig. 3 of the paper by Bohannan \& Walborn (1989).

BAT99-23: This star (MG 9) was recently discovered by Morgan (1999). It is one of the faintest $\mathrm{W}-\mathrm{R}$ stars in the LMC, however this unusual faintness is not intrinsic but due to extinction. The HeII $\lambda 4686$ line is weaker than in most LMC WN3 stars.

BAT99-24 (Brey 19): According to Dopita et al. (1994), there is a large ring nebula in both [OIII] and $\mathrm{H} \alpha$, some $135^{\prime \prime} \times 160^{\prime \prime}$ in diameter, brightest to the southwest.

BAT99-25 (Brey 19a): This W - R star, discovered by Morgan \& Good (1990), lies outside the area searched by Azzopardi \& Breysacher (1980). The object is designated as Brey 19a by Lortet (1991) who erroneously identified it as BE-456. The correct identification is BE-546.
BAT99-26 (Brey 20): Dopita et al. (1994) reported the discovery of a very small ring nebula, $12^{\prime \prime} \times 19^{\prime \prime}$ in diameter, associated with the star. A spectrum of this star is shown in Fig. 1a.

BAT99-27 (Brey 21): The star is located in an apparent association which includes the compact cluster HD 35342, S Doradus (HD 35343) and HDE 269357 (Walborn 1977). According to Smith et al. (1996), CIV $\lambda 5808 \sim \mathrm{HeII}$ $\lambda 5411$ indicates WN5 rather than WN3; a plateau of emission longward of HeII $\lambda 4686$ is unidentified and unusual.

BAT99-28 (Brey 22): It is a confirmed binary system of period $P=14.926$ days (Moffat et al. 1990); a spectrum in the range $4000-7000 \AA$ is shown in Fig. 1 of their paper.

According to Dopita et al. (1994), no nebular features appear to be associated with the star.

BAT99-29 (Brey 23): Niemela et al. (1991) reported that from their systematic search for high excitation nebulae around $\mathrm{W}-\mathrm{R}$ stars in the Magellanic Clouds, no evidence for nebular HeII $\lambda 4686$ emission in the vicinity of the star could be found. Dopita et al. (1994) confirmed that no nebular features are associated with the $\mathrm{W}-\mathrm{R}$ star itself.

According to Morris et al. (1993), the continuum energy distribution of the star can be fitted by a power law of the form $F_{\lambda} \sim \lambda^{-\alpha}$, where $\alpha=2.74$, over the range $1500-9000 \AA$.

BAT99-30 (Brey 24): Koesterke et al. (1991) derived the following values for the "core radius" $R_{*}$, the related effective temperature $T_{*}$, the luminosity $L$ and the mass-loss rate $\dot{M}$ of the star:

$R_{*}=13 R_{\odot} ; T_{*}=33000 \mathrm{~K} ; \log \left(L / L_{\odot}\right)=5.2 ;$ $\log \left(\dot{M} /\left(M_{\odot} \mathrm{yr}^{-1}\right)\right)=-4.5$.

Crowther \& Smith (1997) obtained fairly similar stellar parameters:

$R_{*}=12.6 R_{\odot} ; T_{*}=36400 \mathrm{~K} ; \log \left(L / L_{\odot}\right)=5.40 ;$ $\log \left(\dot{M} /\left(M_{\odot} \mathrm{yr}^{-1}\right)\right)=-4.60$.

According to Morris et al. (1993), the continuum energy distribution of the star can be fitted by a power law of the form $F_{\lambda} \sim \lambda^{-\alpha}$, where $\alpha=2.99$, over the range $1500-9000 \AA$.

BAT99-31 (Brey 25): Niemela et al. (1991) reported that from their systematic search for high excitation nebulae around $\mathrm{W}-\mathrm{R}$ stars in the Magellanic Clouds, no evidence for nebular HeII $\lambda 4686$ emission in the vicinity of the star could be found.

According to Morris et al. (1993), the continuum energy distribution of the star can be fitted by a power law of the form $F_{\lambda} \sim \lambda^{-\alpha}$, where $\alpha=2.74$, over the range $1500-9000 \AA$. 
BAT99-32 (Brey 26): According to Moffat (1989), it is a binary system of period $P=1.9075$ days.

Koesterke et al. (1991) derived the following values for the "core radius" $R_{*}$, the related effective temperature $T_{*}$, the luminosity $L$ and the mass-loss rate $\dot{M}$ of the star:

$R_{*}=17 R_{\odot} ; T_{*}=33000 \mathrm{~K} ; \log \left(L / L_{\odot}\right)=5.5$; $\log \left(\dot{M} /\left(M_{\odot} \mathrm{yr}^{-1}\right)\right)=-4.2$.

Crowther \& Smith (1997) obtained fairly similar stellar parameters:

$R_{*}=16.9 R_{\odot} ; T_{*}=35300 \mathrm{~K} ; \log \left(L / L_{\odot}\right)=5.60 ;$ $\log \left(\dot{M} /\left(M_{\odot} \mathrm{yr}^{-1}\right)\right)=-4.12$, assuming that the visual spectrum of the object is entirely due to the $\mathrm{W}-\mathrm{R}$ star.

According to Morris et al. (1993), the continuum energy distribution of the star can be fitted by a power law of the form $F_{\lambda} \sim \lambda^{-\alpha}$, where $\alpha=3.24$, over the range $1500-9000 \AA$.

Dopita et al. (1994) reported the discovery of a very small ring nebula, $16^{\prime \prime}$ in diameter, which appears as a bright knot in $\mathrm{H} \alpha$ to the east of the star, and as an arc of nebulosity to the south.

A spectrum of this star is shown in Fig. 1b.

BAT99-33: It is remarkable that this star, now classified Ofpe/WN9, was already included in the list of $\mathrm{W}-\mathrm{R}$ stars recognised by Miss Cannon and Miss Payne (cf. Buscombe et al. 1954).

Pasquali et al. (1997) derived the following values for the "core radius" $R_{*}$, the related effective temperature $T_{*}$, the luminosity $L$ and the mass-loss rate $\dot{M}$ of the star:

$R_{*}=39 R_{\odot} ; T_{*}=34800 \mathrm{~K} ; \log \left(L / L_{\odot}\right)=6.3 ;$ $\log \left(\dot{M} /\left(M_{\odot} \mathrm{yr}^{-1}\right)\right)=-4.6$.

According to Morris et al. (1993), the continuum energy distribution of the star can be fitted by a power law of the form $F_{\lambda} \sim \lambda^{-\alpha}$, where $\alpha=2.19$, over the range $1500-9000 \AA$.

Nota et al. (1996) reported the existence of a surrounding nebulosity.

A spectrum of the star in the range $3900-4700 \AA$ is shown in Fig. 4 of the paper by Bohannan \& Walborn (1989).

BAT99-35 (Brey 27): From an analysis of the helium spectrum, Koesterke et al. (1991) have derived the following set of values for the "core radius" $R_{*}$, the related effective temperature $T_{*}$, the luminosity $L$ and the mass-loss rate $\dot{M}$ of the star:

$R_{*}=5.5 R_{\odot} ; T_{*}=39000 \mathrm{~K} ; \log \left(L / L_{\odot}\right)=4.8 ;$ $\log \left(\dot{M} /\left(M_{\odot} \mathrm{yr}^{-1}\right)\right)=-4.6$.

$R_{*}=3.0 R_{\odot} ; T_{*}=90000 \mathrm{~K}$ (adopted); $\log \left(L / L_{\odot}\right)=5.7$; $\log \left(\dot{M} /\left(M_{\odot} \mathrm{yr}^{-1}\right)\right)=-4.3$.

These authors point out that the stellar parameters corresponding to the first entry appear to be unrealistic given the WN3 spectral type of the star; the presence of the HeI $\lambda 5876$ line is questioned.
According to Morris et al. (1993), the continuum energy distribution of the star can be fitted by a power law of the form $F_{\lambda} \sim \lambda^{-\alpha}$, where $\alpha=2.58$, over the range $1500-9000 \AA$.

Possibly a borderline WN4 according to Smith et al. (1996).

BAT99-36 (Brey 29): The CIV $\lambda 5808$ emission line is unusually strong for a WN-type star (Breysacher 1981). A spectrum of this star is shown in Fig. 1a.

Koesterke et al. (1991) derived the following values for the "core radius" $R_{*}$, the related effective temperature $T_{*}$, the luminosity $L$ and the mass-loss rate $\dot{M}$ of the star:

$R_{*}=4.0 R_{\odot} ; T_{*}=47000 \mathrm{~K} ; \log \left(L / L_{\odot}\right)=4.9 ;$ $\log \left(\dot{M} /\left(M_{\odot} \mathrm{yr}^{-1}\right)\right)=-4.3$.

Crowther et al. (1995), found very similar stellar parameters from their standard model A analysis, however, in view of the possible contamination of the $\mathrm{W}-\mathrm{R}$ line spectrum by a companion, they performed a second analysis (model B) which takes into account the binarity. The revised parameters then obtained are as follows:

$R_{*}=3 R_{\odot} ; T_{*}=63000 \mathrm{~K} ; \log \left(L / L_{\odot}\right)=5.0$; $\log \left(\dot{M} /\left(M_{\odot} \mathrm{yr}^{-1}\right)\right)=-4.3$.

According to Morris et al. (1993), the continuum energy distribution of the star can be fitted by a power law of the form $F_{\lambda} \sim \lambda^{-\alpha}$, where $\alpha=2.69$, over the range $1500-9000 \AA$.

Chu \& Lasker (1980) identified DEM 174 as a ring nebula, however, according to Dopita et al. (1994) higher resolution images do not show any shell structure. Niemela et al. (1991) reported that from their systematic search for high excitation nebulae around $\mathrm{W}-\mathrm{R}$ stars in the Magellanic Clouds, no evidence for nebular HeII $\lambda 4686$ emission in the vicinity of the star could be found.

BAT99-37 (Brey 30): Dopita et al. (1994) reported the discovery of a small ring nebula, $26^{\prime \prime} \times 42^{\prime \prime}$ in diameter, extended in the north-south direction.

BAT99-38 (Brey 31): It is a confirmed binary system of period $P=3.03269$ days (Moffat et al. 1990); a spectrum in the range $4000-7000 \AA$ is shown in Fig. 2 of their paper.

Dopita et al. (1994) reported the discovery of a filamentary ring nebula, $33^{\prime \prime}$ in diameter, visible in [OIII]. In $\mathrm{H} \alpha$ a complex nebula in the form of a series of connected loops extends to the south-southwest.

BAT99-39 (Brey 32): It is a confirmed binary system of period $P=1.91674$ days (Moffat et al. 1990); a spectrum in the range $4000-7000 \AA$ is shown in Fig. 3 of their paper. According to Seggewiss et al. (1991), the orbital inclination is too low to allow detectable transparency eclipses. 
BAT99-40 (Brey 33): According to Smith et al. (1996), the $\mathrm{W}-\mathrm{R}$ spectrum is heavily affected by an $\mathrm{O}$ absorption spectrum.

BAT99-41 (Brey 35): According to Morris et al. (1993), the continuum energy distribution of the star can be fitted by a power law of the form $F_{\lambda} \sim \lambda^{-\alpha}$, where $\alpha=2.73$, over the range $1500-9000 \AA$.

BAT99-42 (Brey 34): Seggewiss et al. (1991) remarked that the fairly long spectroscopic period of this $\operatorname{star}(P=$ 30.18 : days) is still poorly known.

Dopita et al. (1994) reported the presence of a clearly defined filamentary ring nebula, $49^{\prime \prime}$ in diameter, brightest in two arcs to the northwest and to the southeast.

According to Smith et al. (1996), CIV $\lambda 5808 \sim \mathrm{HeII}$ $\lambda 5411$ indicates WN5 rather than WN3; a plateau of emission longward of HeII $\lambda 4686$ is unidentified and unusual.

BAT99-43 (Brey 37): From an analysis of the helium spectrum, Koesterke et al. (1991) have derived the following set of values for the "core radius" $R_{*}$, the related effective temperature $T_{*}$, the luminosity $L$ and the mass-loss rate $\dot{M}$ of the star:

$R_{*}=8.5 R_{\odot} ; T_{*}=35000 \mathrm{~K} ; \log \left(L / L_{\odot}\right)=5.0$; $\log \left(\dot{M} /\left(M_{\odot} \mathrm{yr}^{-1}\right)\right)=-4.5$.

$R_{*}=5.1 R_{\odot} ; T_{*}=90000 \mathrm{~K}$ (adopted); $\log \left(L / L_{\odot}\right)=6.2$; $\log \left(\dot{M} /\left(M_{\odot} \mathrm{yr}^{-1}\right)\right)=-4.3$.

These authors point out that the stellar parameters corresponding to the first entry appear to be unrealistic given the spectral type of the star; the presence of the HeI $\lambda 5876$ line is questioned.

Niemela et al. (1991) reported that from their systematic search for high excitation nebulae around $\mathrm{W}-\mathrm{R}$ stars in the Magellanic Clouds, no evidence for nebular HeII $\lambda 4686$ emission in the vicinity of the star could be found. Dopita et al. (1994) reported the possible presence of a very faint, small ring nebula in $[\mathrm{OIII}], 13^{\prime \prime}$ in diameter, and brightest on the east side of the star.

According to Moffat (1998), BAT99-43 is a shortperiod (SB2) binary system.

BAT99-44 (Brey 36): Crowther \& Smith (1997) derived the following values for the "core radius" $R_{*}$, the related effective temperature $T_{*}$, the luminosity $L$ and the mass-loss rate $\dot{M}$ of the star:

$R_{*}=13.4 R_{\odot} ; T_{*}=33700 \mathrm{~K} ; \log \left(L / L_{\odot}\right)=5.32 ;$ $\log \left(\dot{M} /\left(M_{\odot} \mathrm{yr}^{-1}\right)\right)=-4.74$.

According to Morris et al. (1993), the continuum energy distribution of the star can be fitted by a power law of the form $F_{\lambda} \sim \lambda^{-\alpha}$, where $\alpha=3.07$, over the range $1500-9000 \AA$.

BAT99-45: Crowther \& Smith (1997) derived the following values for the "core radius" $R_{*}$, the related effective temperature $T_{*}$, the luminosity $L$ and the mass-loss rate $\dot{M}$ of the star:

$R_{*}=30.3 R_{\odot} ; T_{*}=29200 \mathrm{~K} ; \log \left(L / L_{\odot}\right)=5.78 ;$ $\log \left(\dot{M} /\left(M_{\odot} \mathrm{yr}^{-1}\right)\right)=-4.30$.

Pasquali et al. (1997) obtained for these parameters:

$R_{*}=32 R_{\odot} ; T_{*}=32100 \mathrm{~K} ; \log \left(L / L_{\odot}\right)=6.0 ;$ $\log \left(\dot{M} /\left(M_{\odot} \mathrm{yr}^{-1}\right)\right)=-4.3$.

A spectrum of the star in the range $3900-4700 \AA$ is shown in Fig. 3 of the paper by Bohannan \& Walborn (1989).

According to van Genderen \& Sterken (1996), the observed light variations of BAT99-45 show a timescale of 1-2 years and have an amplitude of $0.3-0.5 \mathrm{mag}$, which points to an LBV near minimum light. The colour variation is also typical for an LBV.

BAT99-46 (Brey 38): According to Morris et al. (1993), the continuum energy distribution of the star can be fitted by a power law of the form $F_{\lambda} \sim \lambda^{-\alpha}$, where $\alpha=2.03$, over the range $1500-9000 \AA$.

BAT99-48 (Brey 40): Koesterke et al. (1991) derived the following values for the "core radius" $R_{*}$, the related effective temperature $T_{*}$, the luminosity $L$ and the mass-loss rate $\dot{M}$ of the star:

$R_{*}=2.9 R_{\odot} ; T_{*}=57000 \mathrm{~K} ; \log \left(L / L_{\odot}\right)=4.9 ;$ $\log \left(\dot{M} /\left(M_{\odot} \mathrm{yr}^{-1}\right)\right)=-4.5$.

According to Morris et al. (1993), the continuum energy distribution of the star can be fitted by a power law of the form $F_{\lambda} \sim \lambda^{-\alpha}$, where $\alpha=2.52$, over the range $1500-9000 \AA$.

BAT99-49 (Brey 40a): Niemela (1991) found this object to be a double-lined binary with an elliptic orbit $(e=0.41)$ of period about 34 days. Niemela et al. (1991) also reported the detection of nebular HeII $\lambda 4686$ line emission over a region having an extent of $70^{\prime \prime}$. According to Dopita et al. (1994), the star is surrounded by a circular ring nebula in $\mathrm{H} \alpha$, some $40^{\prime \prime}$ in diameter.

BAT99-52 (Brey 43): According to Morris et al. (1993), the continuum energy distribution of the star can be fitted by a power law of the form $F_{\lambda} \sim \lambda^{-\alpha}$, where $\alpha=3.18$, over the range $1500-9000 \AA$.

BAT99-53 (Brey 44): Niemela et al. (1991) reported that from their systematic search for high excitation nebulae around $\mathrm{W}-\mathrm{R}$ stars in the Magellanic Clouds, no evidence for nebular HeII $\lambda 4686$ emission in the vicinity of the star could be found. According to Niemela (1995), this star shows radial velocity variations of emission and absorption lines in opposite sense indicating a long-period binary.

BAT99-54 (Brey 44a): Crowther \& Smith (1997) derived the following values for the "core radius" $R_{*}$, the related effective temperature $T_{*}$, the luminosity $L$ and the 
mass-loss rate $\dot{M}$ of the star:

$R_{*}=20.0 R_{\odot} ; T_{*}=30900 \mathrm{~K} ; \log \left(L / L_{\odot}\right)=5.52$; $\log \left(\dot{M} /\left(M_{\odot} \mathrm{yr}^{-1}\right)\right)=-5.01$.

BAT99-55: Crowther \& Smith (1997) derived, from their model A analysis, the following values for the "core radius" $R_{*}$, the related effective temperature $T_{*}$, the luminosity $L$ and the mass-loss rate $\dot{M}$ of the star:

$R_{*}=32.6 R_{\odot} ; T_{*}=27900 \mathrm{~K} ; \log \left(L / L_{\odot}\right)=5.76$; $\log \left(\dot{M} /\left(M_{\odot} \mathrm{yr}^{-1}\right)\right)=-4.87$.

Pasquali et al. (1997) obtained for these parameters:

$R_{*}=35 R_{\odot} ; T_{*}=30900 \mathrm{~K} ; \log \left(L / L_{\odot}\right)=6.0$; $\log \left(\dot{M} /\left(M_{\odot} \mathrm{yr}^{-1}\right)\right)=-4.6$.

A spectrum of the star in the range $3900-4700 \AA$ is shown in Fig. 3 of the paper by Bohannan \& Walborn (1989).

According to Nota et al. (1996), the radial velocity map of the circumstellar nebula $\left(7.7^{\prime \prime} \times 8.6^{\prime \prime}\right.$ in size, and showing a bipolar morphology) is clearly consistent with a hollow shell expanding at $\sim 30 \mathrm{~km} \mathrm{~s}^{-1}$.

BAT99-56 (Brey 46): This star is noted as variable by Feitzinger \& Isserstedt (1983).

Niemela et al. (1991) reported that from their systematic search for high excitation nebulae around $\mathrm{W}-\mathrm{R}$ stars in the Magellanic Clouds, no evidence for nebular HeII $\lambda 4686$ emission in the vicinity of the star could be found.

According to Morris et al. (1993), the continuum energy distribution of the star can be fitted by a power law of the form $F_{\lambda} \sim \lambda^{-\alpha}$, where $\alpha=3.04$, over the range $1500-9000 \AA$.

BAT99-58 (Brey 47): Koesterke et al. (1991) derived the following values for the "core radius" $R_{*}$, the related effective temperature $T_{*}$, the luminosity $L$ and the mass-loss rate $\dot{M}$ of the star:

$R_{*}=10 R_{\odot} ; T_{*}=34000 \mathrm{~K} ; \log \left(L / L_{\odot}\right)=5.1$; $\log \left(\dot{M} /\left(M_{\odot} \mathrm{yr}^{-1}\right)\right)=-4.8$.

Crowther \& Smith (1997) obtained fairly similar stellar parameters:

$R_{*}=11.4 R_{\odot} ; T_{*}=35700 \mathrm{~K} ; \log \left(L / L_{\odot}\right)=5.28$; $\log \left(\dot{M} /\left(M_{\odot} \mathrm{yr}^{-1}\right)\right)=-4.70$.

A spectrum of the star in the range $4200-5300 \AA$ is shown in Fig. 1 of the paper by Morgan et al. (1992).

BAT99-59 (Brey 48): From an analysis of the helium spectrum, Koesterke et al. (1991) have derived the following set of values for the "core radius" $R_{*}$, the related effective temperature $T_{*}$, the luminosity $L$ and the mass-loss rate $\dot{M}$ of the star:

$R_{*}=15 R_{\odot} ; T_{*}=40000 \mathrm{~K} ; \log \left(L / L_{\odot}\right)=5.7$; $\log \left(\dot{M} /\left(M_{\odot} \mathrm{yr}^{-1}\right)\right)=-4.4$.

$R_{*}=8.2 R_{\odot} ; T_{*}=90000 \mathrm{~K}$ (adopted); $\log \left(L / L_{\odot}\right)=6.6$; $\log \left(\dot{M} /\left(M_{\odot} \mathrm{yr}^{-1}\right)\right)=-4.2$.

These authors point out that the HeI $\lambda 5876$ emission is below safe detection, therefore the determined temperature (first entry) corresponds to the limit below which the HeI $\lambda 5876$ line would start to become visible.

A superb ring nebula studied by Chu \& Lasker (1980). According to Dopita et al. (1994), the inner filamentary ring of swept-up matter has a diameter of $80^{\prime \prime} \times 97^{\prime \prime}$, while the outer, more diffuse parts can be traced out to a distance of $115^{\prime \prime}$ from the exciting star.

BAT99-60 (Brey 49): Niemela et al. (1991) reported that from their systematic search for high excitation nebulae around $\mathrm{W}-\mathrm{R}$ stars in the Magellanic Clouds, no evidence for nebular HeII $\lambda 4686$ emission in the vicinity of the star could be found.

BAT99-61 (Brey 50): According to Morris et al. (1993), the continuum energy distribution of the star can be fitted by a power law of the form $F_{\lambda} \sim \lambda^{-\alpha}$, where $\alpha=2.98$, over the range $1500-9000 \AA$.

BAT99-62 (Brey 51): A fine partial ring nebula, of diameter $\sim 45^{\prime \prime}$ (Dopita et al. 1994), appearing as a bright arc to the north of the star was discovered by Pakull (1991).

Possibly a borderline WN4 according to Smith et al. (1996).

BAT99-63 (Brey 52): Cowley et al. (1984) found no evidence for binary motion, but noted the high velocity of the star, possibly a "runaway".

From an analysis of the helium spectrum, Koesterke et al. (1991) have derived the following set of values for the "core radius" $R_{*}$, the related effective temperature $T_{*}$, the luminosity $L$ and the mass-loss rate $\dot{M}$ of the star:

$R_{*}=5.9 R_{\odot} ; T_{*}=35000 \mathrm{~K} ; \log \left(L / L_{\odot}\right)=4.6 ;$ $\log \left(\dot{M} /\left(M_{\odot} \mathrm{yr}^{-1}\right)\right)=-4.9$.

$R_{*}=3.1 R_{\odot} ; T_{*}=90000 \mathrm{~K}$ (adopted); $\log \left(L / L_{\odot}\right)=5.8 ;$ $\log \left(\dot{M} /\left(M_{\odot} \mathrm{yr}^{-1}\right)\right)=-4.7$.

These authors point out that the HeI $\lambda 5876$ emission is below safe detection, therefore the determined temperature (first entry) corresponds to the limit below which the HeI $\lambda 5876$ line would start to become visible.

A ring nebula was claimed for this object by $\mathrm{Chu} \&$ Lasker (1980). According to Dopita et al. (1994), they identified the bright HII region to the northeast as a ring nebula, whereas it is apparent that the true nebula is a fine filamentary ring $65^{\prime \prime} \times 88^{\prime \prime}$ in diameter.

BAT99-64 (Brey 53): Niemela et al. (1991) reported that from their systematic search for high excitation nebulae around $\mathrm{W}-\mathrm{R}$ stars in the Magellanic Clouds, no evidence for nebular HeII $\lambda 4686$ emission in the vicinity of the star could be found.

BAT99-65 (Brey 55): Dopita et al. (1994) reported the discovery of a ring nebula elongated in the northwest to southeast direction, $20^{\prime \prime} \times 29^{\prime \prime}$ in diameter. The outer 
filamentary shell has a fainter shell inside of diameter $12^{\prime \prime}$.

BAT99-67 (Brey 56): This star is noted as variable by Feitzinger \& Isserstedt (1983). According to Smith et al. (1996) it is a binary with an OB component.

From an analysis of the helium spectrum, Koesterke et al. (1991) have derived the following set of values for the "core radius" $R_{*}$, the related effective temperature $T_{*}$, the luminosity $L$ and the mass-loss rate $\dot{M}$ of the star:

$R_{*}=16 R_{\odot} ; T_{*}=32000 \mathrm{~K} ; \log \left(L / L_{\odot}\right)=5.4$; $\log \left(\dot{M} /\left(M_{\odot} \mathrm{yr}^{-1}\right)\right)=-4.3$.

$R_{*}=8.9 R_{\odot} ; T_{*}=90000 \mathrm{~K}$ (adopted); $\log \left(L / L_{\odot}\right)=6.7$; $\log \left(\dot{M} /\left(M_{\odot} \mathrm{yr}^{-1}\right)\right)=-4.6$.

These authors point out that the HeI $\lambda 5876$ emission is below safe detection, therefore the determined temperature (first entry) corresponds to the limit below which the HeI $\lambda 5876$ line would start to become visible.

According to Dopita et al. (1994), there is a flocculent nebulosity associated with the star, $40^{\prime \prime}$ in diameter. This is contained within the X-ray superbubble $0536-692$ (Mathewson et al. 1985), which is part of N 157.

BAT99-68 (Brey 58): This star is located in the tight cluster $\alpha$ in the western part of LH90. A spectrum in the range $3800-6800 \AA$ is shown in Fig. $2 \mathrm{a}$ of the paper by Testor et al. (1993) who derived a WN6-7 spectral type. The star is classified Of by Smith et al. (1996).

BAT99-69: This star is located in the tight cluster $\alpha$ in the western part of LH90. A spectrum in the range $3800-6800 \AA$ is shown in Fig. 2b of the paper by Testor et al. (1993).

BAT99-71 (Brey 60): Smith et al. (1996) pointed out that the HeII emission lines are weak and very narrow for a WN3; they classified the star as peculiar.

BAT99-76 (Brey 64): A spectrum of the star in the range $3900-4700 \AA$ is shown in Fig. 3 of the paper by Bohannan \& Walborn (1989), and in the range $4000-7500 \AA$ in Fig. 1b of the present paper.

Koesterke et al. (1991) derived the following values for the "core radius" $R_{*}$, the related effective temperature $T_{*}$, the luminosity $L$ and the mass-loss rate $\dot{M}$ of the star:

$R_{*}=19 R_{\odot} ; T_{*}=30000 \mathrm{~K} ; \log \left(L / L_{\odot}\right)=5.4$; $\log \left(\dot{M} /\left(M_{\odot} \mathrm{yr}^{-1}\right)\right)=-4.6$.

Crowther et al. (1995) obtained fairly similar stellar parameters:

$R_{*}=20.8 R_{\odot} ; T_{*}=30600 \mathrm{~K} ; \log \left(L / L_{\odot}\right)=5.54 ;$ $\log \left(\dot{M} /\left(M_{\odot} \mathrm{yr}^{-1}\right)\right)=-4.65$.

Pasquali et al. (1997) obtained for these parameters:

$R_{*}=18 R_{\odot} ; T_{*}=38700 \mathrm{~K} ; \log \left(L / L_{\odot}\right)=5.8$; $\log \left(\dot{M} /\left(M_{\odot} \mathrm{yr}^{-1}\right)\right)=-4.7$.

According to Morris et al. (1993), the continuum energy distribution of the star can be fitted by a power law of the form $F_{\lambda} \sim \lambda^{-\alpha}$, where $\alpha=3.17$, over the range $1500-9000 \AA$.

Nota et al. (1996) reported the existence of a surrounding shell which expands at a velocity of $\sim 30 \mathrm{~km} \mathrm{~s}^{-1}$. According to Pasquali, Nota \& Clampin (1998), two diffuse arches define the shell of diameter $13^{\prime \prime}$, which is not of stellar origin and probably represents the relic of the interstellar bubble blown by the star during its $\mathrm{O}$ main-sequence phase.

BAT99-77 (Brey 65): This star is located in the tight cluster $\beta=$ HD 269828 in the western part of LH90. Moffat (1989) showed that the star is a binary system with a period $P=3.0032$ days. Spectral variations in the range $4000-4800 \AA$ are illustrated in Fig. 3 of the paper by Heydari-Malayeri et al. (1993).

According to Morris et al. (1993), the continuum energy distribution of the star can be fitted by a power law of the form $F_{\lambda} \sim \lambda^{-\alpha}$, where $\alpha=3.46$, over the range $1500-9000 \AA$.

BAT99-78 (Brey 65b): This star is located in the tight cluster $\beta=$ HD 269828 in the western part of LH90. A spectrum in the range $3700-5500 \AA$ is shown in Fig. 5 of the paper by Testor \& Schild (1990).

The object is designated as Brey 65b by Lortet (1991).

The composite nature of this star was recognized by Heydari-Malayeri et al. (1993), and confirmed by HST/WFC observations (Walborn et al. 1995) which further resolved the object into five components. According to Walborn et al. (1998), the WN star is definitely component 5C. The HST image of BAT99-78 is reproduced in Fig. 8.

BAT99-79 (Brey 57): A spectrum of the star in the range $4000-4800 \AA$ is shown in Fig. 3 of the paper by HeydariMalayeri et al. (1993).

According to Morris et al. (1993), the continuum energy distribution of the star can be fitted by a power law of the form $F_{\lambda} \sim \lambda^{-\alpha}$, where $\alpha=3.88$, over the range $1500-9000 \AA$.

BAT99-80 (Brey 65c): This star is located in the tight cluster $\beta=$ HD 269828 in LH90 and its spectrum, in the range $3700-5500 \AA$, is shown in Fig. 5 of the paper by Testor \& Schild (1990).

The object is designated as Brey 65c by Lortet (1991).

BAT99-81 (Brey 65a): This W - R star was discovered serendipitously by Cowley et al. (1984) who reported a significant decrease in intensity of the emission spectrum on one night (JD 2445732).

According to Morris et al. (1993), the continuum energy distribution of the star can be fitted by a power law of the form $F_{\lambda} \sim \lambda^{-\alpha}$, where $\alpha=3.10$, over the range $1500-9000 \AA$. 
BAT99-82 (Brey 66): Niemela et al. (1991) reported that from their systematic search for high excitation nebulae around $\mathrm{W}-\mathrm{R}$ stars in the Magellanic Clouds, no evidence for nebular HeII $\lambda 4686$ emission in the vicinity of the star could be found.

A spectrum of the star in the range $4500-6700 \AA$ is shown in Fig. 1 of the paper by Morgan, Watson \& Parker (1992).

BAT99-83: This star is now categorized as a Luminous Blue Variable. According to Nota et al. (1991), it is a very luminous $\left(L \approx 10^{6} L_{\odot}\right)$ star with an initial mass between $60 M_{\odot}$ and $100 M_{\odot}$.

Smith et al. (1998) found that the circumstellar environment of BAT99-83 reveals two discrete ejections in the mass loss history of the central star. There is an inner expanding shell, with a radius of $0.6 \mathrm{pc}$, an expansion velocity of $29 \mathrm{~km} \mathrm{~s}^{-1}$, and a dynamical age of $210^{4}$ years. There is also material beyond the bright inner nebula that may represent an outer expanding shell with a radius of $1.9 \mathrm{pc}$ and a dynamical age of $710^{4}$ years.

BAT99-84 (Brey 68): According to Bartzakos (1998), the absorption-line spectrum might arise in a visual $\mathrm{OB}$ companion, therefore the star is considered as "almost certainly single".

BAT99-85 (Brey 67): According to Bartzakos (1998), the absorption-line spectrum might arise in a visual $\mathrm{OB}$ companion, therefore the star is considered as "almost certainly single".

BAT99-87 (Brey 70): According to Bartzakos (1998), the absorption-line spectrum might arise in a visual $\mathrm{OB}$ companion, therefore the star is considered as "almost certainly single".

BAT99-88 (Brey 70a): According to Lortet et al. (1991), this star is outstanding by its high luminosity $\left(M_{\mathrm{bol}} \approx-10.0\right)$, high reddening, strong and wide lines, and intense CIV $\lambda 5808$ emission.

BAT99-89 (Brey 71): Crowther \& Smith (1997) derived the following values for the "core radius" $R_{*}$, the related effective temperature $T_{*}$, the luminosity $L$ and the mass-loss rate $\dot{M}$ of the star:

$R_{*}=9.4 R_{\odot} ; T_{*}=39400 \mathrm{~K} ; \log \left(L / L_{\odot}\right)=5.28 ;$ $\log \left(\dot{M} /\left(M_{\odot} \mathrm{yr}^{-1}\right)\right)=-4.35$.

According to Dopita et al. (1994), a dust arc - possibly a swept-up shell - appears $20^{\prime \prime}$ to the north of the star in projection against the bright HII region.

BAT99-90 (Brey 74): A spectrum of the star in the range $4500-6700 \AA$ is shown in Fig. 1 of the paper by Morgan et al. (1992).

BAT99-91 (Brey 73): This star is a complex multiple system in LH99 and was resolved into 12 components by Testor et al. (1988), who achieved $0.25^{\prime \prime}$ resolution. HST/WFC observations of the object (Walborn et al. 1995) further resolved into four components each the two brightest components, Nos. 1, (the WN) and 2, identified by Testor et al. (1988). According to Walborn et al. (1998), the WN star is definitely component 1A. The HST image of BAT99-91 is reproduced in Fig. 10.

BAT99-92 (Brey 72): This star is noted as variable in $V$ by Feitzinger \& Isserstedt (1983). Although the W - R component is generally classified $\mathrm{WN}$, the width of the HeII $\lambda 4686$ emission and the strength of the CIV $\lambda 5808$ line probably explain that a WC type has sometimes been assigned as well. The spectral type given in Table 2 is the one determined by Moffat \& Seggewiss (1986) who argued that, unless one of the star has a very peculiar mass, the WN6 component must be orbiting a third, unseen star ( $P=4.3092$ days), which is most likely a normal luminosity $\mathrm{O}$ star of moderate mass, similar to the mass of the WN6 star. Seggewiss et al. (1991) remarked, however, that the light curve shows no convincing evidence for variation with the $\mathrm{W}-\mathrm{R}$ star's orbital period.

Niemela et al. (1991) reported that from their systematic search for high excitation nebulae around $\mathrm{W}-\mathrm{R}$ stars in the Magellanic Clouds, no evidence for nebular HeII $\lambda 4686$ emission in the vicinity of the star could be found.

BAT99-93 (Brey 74a): The object is designated as Brey 74a by Lortet (1991).

BAT99-94 (Brey 85): Breysacher (1981) classified this star as peculiar because of the unusually broad emission lines.

Koesterke et al. (1991) derived the following values for the "core radius" $R_{*}$, the related effective temperature $T_{*}$, the luminosity $L$ and the mass-loss rate $\dot{M}$ of the star:

$R_{*}=1.8 R_{\odot} ; T_{*}=90000 \mathrm{~K} ; \log \left(L / L_{\odot}\right)=5.3 ;$ $\log \left(\dot{M} /\left(M_{\odot} \mathrm{yr}^{-1}\right)\right)=-4.0$.

Niemela et al. (1991) reported that from their systematic search for high excitation nebulae around $\mathrm{W}-\mathrm{R}$ stars in the Magellanic Clouds, no evidence for nebular HeII $\lambda 4686$ emission in the vicinity of the star could be found.

BAT99-95 (Brey 80): Crowther \& Smith (1997) derived the following values for the "core radius" $R_{*}$, the related effective temperature $T_{*}$, the luminosity $L$ and the massloss rate $\dot{M}$ of the star: 
$R_{*}=15.2 R_{\odot} ; T_{*}=36100 \mathrm{~K} ; \log \left(L / L_{\odot}\right)=5.55$; $\log \left(\dot{M} /\left(M_{\odot} \mathrm{yr}^{-1}\right)\right)=-3.70$.

Located in the vicinity of the core of 30 Doradus, BAT9995 has a peculiar spectral appearance among the WNL stars with strong, broad non-Gaussian profiles.

According to Dopita et al. (1994), this star does not appear to be associated with ring features.

BAT99-96 (Brey 81): Crowther \& Smith (1997) derived the following values for the "core radius" $R_{*}$, the related effective temperature $T_{*}$, the luminosity $L$ and the mass-loss rate $\dot{M}$ of the star:

$R_{*}=27.0 R_{\odot} ; T_{*}=32400 \mathrm{~K} ; \log \left(L / L_{\odot}\right)=5.86$; $\log \left(\dot{M} /\left(M_{\odot} \mathrm{yr}^{-1}\right)\right)=-4.02$.

These authors noted that, relative to the other LMC WN8 stars, BAT99-96 shows a high luminosity and very low hydrogen content of $\mathrm{H} / \mathrm{He} \sim 0.2$. According to Dopita et al. (1994), this star does not appear to be associated with ring features.

BAT99-98 (Brey 79): Star located near the core of 30 Doradus.

BAT99-99 (Brey 78): Star located near the core of 30 Doradus.

BAT99-100 (Brey 75): Star located near the core of 30 Doradus. Crowther \& Smith (1997) derived the following values for the "core radius" $R_{*}$, the related effective temperature $T_{*}$, the luminosity $L$ and the mass-loss rate $\dot{M}$ of the star:

$R_{*}=25.9 R_{\odot} ; T_{*}=33100 \mathrm{~K} ; \log \left(L / L_{\odot}\right)=5.86$; $\log \left(\dot{M} /\left(M_{\odot} \mathrm{yr}^{-1}\right)\right)=-4.11$.

BAT99-101+102+103 (Brey 87): According to Moffat et al. (1987), the visual multiple system R 140, in the 30 Doradus nebula, contains at least three $\mathrm{W}-\mathrm{R}$ stars, one of type WC5, the others of type WNL. BAT99-101 $\equiv$ R 140a1 (northern component of R 140a) and BAT99-102 $\equiv \mathrm{R} 140 \mathrm{a} 2$ (southern component of $\mathrm{R} 140 \mathrm{a}$ ) are not resolved on the finding chart (Fig. 12). One of the WNL components (BAT99-102) is found to be a close spectroscopic binary with a period of 2.76 days.

Dopita et al. (1994) noted that the system does not appear to be associated with ring features.

BAT99-104 (Brey 76): Star located near the core of 30 Doradus. In the notation introduced by Melnick (1985) and refined by Parker (1993), the object is subdivided into two components: $\mathrm{Mk} 37 \mathrm{Wa}$ and $\mathrm{Mk}$ 37Wb. According to Massey \& Hunter (1998), the W - R spectrum originates from $\mathrm{Mk} 37 \mathrm{~Wb}$.

BAT99-105 (Brey 77): Star located near the core of 30 Doradus. Heap et al. (1991) derived the following properties for this star: $T_{\text {eff }}=42500 \mathrm{~K} ; R=26 R_{\odot}$; $\log \left(L / L_{\odot}\right)=6.36 ; \log \left(\dot{M} /\left(M_{\odot} \mathrm{yr}^{-1}\right)\right)=-5.4$.

These authors noted that with a present mass of $100 M_{\odot}$, BAT99-105 is one of the most luminous and massive stars known. A detailed NLTE analysis of this star has been presented by de Koter et al. (1997).

BAT99-107 (Brey 86): Hyland et al. (1978) detected an infrared excess $(V-K)_{0}=1.41$ for this star, which is much larger than normally found in galactic WN stars.

According to Moffat (1989), this object, which exhibits a "nonpure" $\mathrm{W}-\mathrm{R}$ spectrum, is a spectroscopic binary. It appears to have been responsible for producing a very bright and fine ring nebula, $49^{\prime \prime} \times 55^{\prime \prime}$ in diameter, brightest on the east and extending to the northwest (Dopita et al. 1994).

BAT99-106 $+\mathbf{1 0 8}+\mathbf{1 0 9}+\mathbf{1 1 0}+\mathbf{1 1 1}+\mathbf{1 1 2}$ (Brey 82): Central cluster of 30 Dor. Speckle interferometric observations (Weigelt \& Baier 1985) and later HST/FOC images (Weigelt et al. 1991) have resolved R 136a - the bright, diffuse central object - into a minimum of eight components (R 136a1 $\rightarrow \mathrm{a} 8)$, all within $1^{\prime \prime}$.

From HST spectroscopy, Crowther \& Dessart (1998) derived the following values for the "core radius" $R_{*}$, the related effective temperature $T_{*}$, the luminosity $L$ and the mass-loss rate $\dot{M}$ of the stars:

- BAT99-108 (R 136a1): $R_{*}=20 R_{\odot} ; T_{*}=45000 \mathrm{~K}$; $\log \left(L / L_{\odot}\right)=6.18$

$\log \left(\dot{M} /\left(M_{\odot} \mathrm{yr}^{-1}\right)\right)=-4.43$

- BAT99-109 (R 136a2): $R_{*}=19 R_{\odot} ; T_{*}=46000 \mathrm{~K}$; $\log \left(L / L_{\odot}\right)=6.16$

$\log \left(M /\left(M_{\odot} \mathrm{yr}^{-1}\right)\right)=-4.57$.

- BAT99-106 (R 136a3): $R_{*}=19 R_{\odot} ; T_{*}=44000 \mathrm{~K}$; $\log \left(L / L_{\odot}\right)=6.07$ $\log \left(M /\left(M_{\odot} \mathrm{yr}^{-1}\right)\right)=-4.27$.

From HST observations (WFPC photometry and GHRS spectroscopy), Heap et al. (1994) derived the following properties for BAT99-110 (R 136a5) : $T_{\text {eff }}=42500 \mathrm{~K} ; R=16.4 R_{\odot} ; \log \left(L / L_{\odot}\right)=5.90 ;$ $M \approx 50 M_{\odot}$. These authors noted that the observed mass-loss rate, $\log \left(\dot{M} /\left(M_{\odot} \mathrm{yr}^{-1}\right)\right)=-4.74$, is an order of magnitude higher than assumed by current stellar evolutionary models.

A detailed NLTE analysis of stars in R 136a has been presented by de Koter et al. (1997).

BAT99-113: Star located near the core of 30 Doradus.

BAT99-114: Star located near the core of 30 Doradus.

BAT99-115 (Brey 83): Star located near the core of 30 Doradus. In the notation introduced by Melnick (1985) and refined by Parker (1993), the object is subdivided into three components: $\mathrm{Mk} 33 \mathrm{Sa}, \mathrm{Mk} 33 \mathrm{Sb}$ and $\mathrm{Mk}$ 33Sc. The $\mathrm{W}-\mathrm{R}$ star corresponds to Mk 33Sb. 
BAT99-116 (Brey 84): Star located near the core of 30 Doradus.

BAT99-117 (Brey 88): From an analysis of the helium spectrum, Koesterke et al. (1991) have derived the following set of values for the "core radius" $R_{*}$, the related effective temperature $T_{*}$, the luminosity $L$ and the mass-loss rate $\dot{M}$ of the star:

$R_{*}=17 R_{\odot} ; T_{*}=33000 \mathrm{~K} ; \log \left(L / L_{\odot}\right)=5.5$; $\log \left(\dot{M} /\left(M_{\odot} \mathrm{yr}^{-1}\right)\right)=-4.5$.

$R_{*}=9.2 R_{\odot} ; T_{*}=90000 \mathrm{~K}$ (adopted); $\log \left(L / L_{\odot}\right)=6.7$; $\log \left(\dot{M} /\left(M_{\odot} \mathrm{yr}^{-1}\right)\right)=-4.7$.

These authors point out that the HeI $\lambda 5876$ emission is below safe detection, therefore the determined temperature (first entry) corresponds to the limit below which the HeI $\lambda 5876$ line would start to become visible.

Dopita et al. (1994) noted that this star is contained within the X-ray-emitting supershell on the northeast side of 30 Dor.

According to Smith et al. (1996), strong Balmer absorption lines are present in the spectrum, but no helium absorption lines.

BAT99-118 (Brey 89): Crowther \& Dessart (1998) derived the following values for the "core radius" $R_{*}$, the related effective temperature $T_{*}$, the luminosity $L$ and the mass-loss rate $\dot{M}$ of the star:

$R_{*}=36 R_{\odot} ; T_{*}=37000 \mathrm{~K} ; \log \left(L / L_{\odot}\right)=6.34 ;$ $\log \left(\dot{M} /\left(M_{\odot} \mathrm{yr}^{-1}\right)\right)=-3.82$.

According to Morris et al. (1993), the continuum energy distribution of the star can be fitted by a power law of the form $F_{\lambda} \sim \lambda^{-\alpha}$, where $\alpha=2.93$, over the range $1500-9000 \AA$.

Dopita et al. (1994) noted that this star is contained within the X-ray-emitting supershell on the northeast side of 30 Dor.

BAT99-119 (Brey 90): According to Moffat (1989), it is a binary system of period $P=25.17$ : days.

Crowther \& Smith (1997) derived the following values for the "core radius" $R_{*}$, the related effective temperature $T_{*}$, the luminosity $L$ and the mass-loss rate $\dot{M}$ of the star: $R_{*}=39.5 R_{\odot} ; T_{*}=31800 \mathrm{~K} ; \log \left(L / L_{\odot}\right)=6.16$; $\log \left(\dot{M} /\left(M_{\odot} \mathrm{yr}^{-1}\right)\right)=-3.84$.

These stellar parameters compare closely with those of BAT99-118, its neighbour in 30 Doradus.

According to Morris et al. (1993), the continuum energy distribution of the star can be fitted by a power law of the form $F_{\lambda} \sim \lambda^{-\alpha}$, where $\alpha=2.79$, over the range $1500-9000 \AA$.

BAT99-120 (Brey 91): Dopita et al. (1994) reported the discovery of a very large filamentary and "cellular" ring nebula, $\sim 240^{\prime \prime}$ in diameter.

Possibly a post-LBV according to Crowther et al. (1995) who derived the following stellar parameters:

$R_{*}=26.3 R_{\odot} ; T_{*}=30000 \mathrm{~K} ; \log \left(L / L_{\odot}\right)=5.70 ;$ $\log \left(\dot{M} /\left(M_{\odot} \mathrm{yr}^{-1}\right)\right)=-4.51$.

Pasquali et al. (1997) obtained for these parameters:

$R_{*}=23 R_{\odot} ; T_{*}=38900 \mathrm{~K} ; \log \left(L / L_{\odot}\right)=6.0$; $\log \left(\dot{M} /\left(M_{\odot} \mathrm{yr}^{-1}\right)\right)=-4.6$.

Nota et al. (1996) reported the presence of strong nebular emissions in the spectrum of BAT99-120 pointing out, however, that the association of these lines with the star is less obvious. This star is located only $3^{\prime \prime}$ west of Sk -69 249 .

BAT99-122 (Brey 92): Hyland et al. (1978) detected an infrared excess $(V-K)_{0}=2.10$ for this star, which is much larger than normally found in galactic WN stars.

According to Morris et al. (1993), the continuum energy distribution of the star can be fitted by a power law of the form $F_{\lambda} \sim \lambda^{-\alpha}$, where $\alpha=2.79$, over the range $1500-9000 \AA$.

Dopita et al. (1994) noted that this star is contained within the X-ray-emitting supershell on the northeast side of 30 Dor.

BAT99-123 (Brey 93): According to Melnick \& HeydariMalayeri (1991), the star is embedded in a bright HII region having a very complex structure in [OIII]. The absence of HeII $\lambda 4686$ nebular emission in the ring nebula around the star was noted by Pakull (1991). A spectrum of this star is shown in Fig. 1c.

BAT99-124 (Brey 93a): Niemela et al. (1991) reported that from their systematic search for high excitation nebulae around $\mathrm{W}-\mathrm{R}$ stars in the Magellanic Clouds, no evidence for nebular HeII $\lambda 4686$ emission in the vicinity of the star could be found.

Dopita et al. (1994) reported the discovery of a small asymmetric ring nebula, $\sim 20^{\prime \prime}$ in diameter, brightest to the east.

BAT99-126 (Brey 95): This star is noted as variable in $V$ by Feitzinger \& Isserstedt (1983).

Niemela et al. (1991) reported that from their systematic search for high excitation nebulae around $\mathrm{W}-\mathrm{R}$ stars in the Magellanic Clouds, no evidence for nebular HeII $\lambda 4686$ emission in the vicinity of the star could be found.

BAT99-130: Crowther \& Smith (1997) derived the following values for the "core radius" $R_{*}$, the related effective temperature $T_{*}$, the luminosity $L$ and the mass-loss rate $\dot{M}$ of the star:

$R_{*}=28.4 R_{\odot} ; T_{*}=28400 \mathrm{~K} ; \log \left(L / L_{\odot}\right)=5.67 ;$ $\log \left(\dot{M} /\left(M_{\odot} \mathrm{yr}^{-1}\right)\right)=-4.80$.

A spectrum of the star in the range $3900-4700 \AA$ is shown in Fig. 3 of the paper by Bohannan \& Walborn (1989). 
BAT99-132 (Brey 99): Niemela et al. (1991) reported that from their systematic search for high excitation nebulae around $\mathrm{W}-\mathrm{R}$ stars in the Magellanic Clouds, no evidence for nebular HeII $\lambda 4686$ emission in the vicinity of the star could be found.

According to Morris et al. (1993), the continuum energy distribution of the star can be fitted by a power law of the form $F_{\lambda} \sim \lambda^{-\alpha}$, where $\alpha=1.96$, over the range $1500-9000 \AA$.

BAT99-133: Walborn (1982b) considered this star to be a spectroscopic twin to R 127 (BAT99-83) in minimum.

Crowther \& Smith (1997) derived the following values for the "core radius" $R_{*}$, the related effective temperature $T_{*}$, the luminosity $L$ and the mass-loss rate $\dot{M}$ of the star:

$R_{*}=33.3 R_{\odot} ; T_{*}=28300 \mathrm{~K} ; \log \left(L / L_{\odot}\right)=5.76 ;$ $\log \left(\dot{M} /\left(M_{\odot} \mathrm{yr}^{-1}\right)\right)=-4.96$,

and obtained, after nebular subtraction, a relatively low hydrogen content of $\mathrm{H} / \mathrm{He} \sim 1.2$.

Pasquali et al. (1997) obtained for these parameters:

$R_{*}=28 R_{\odot} ; T_{*}=36100 \mathrm{~K} ; \log \left(L / L_{\odot}\right)=6.1$; $\log \left(\dot{M} /\left(M_{\odot} \mathrm{yr}^{-1}\right)\right)=-4.7$.

Pasquali et al. (1998) have spatially resolved the circumstellar nebula associated wit BAT99-133. The nebula has the appearance of a shell, 7.6" in diameter, having a velocity expansion of $28 \mathrm{~km} \mathrm{~s}^{-1}$, which indicates a dynamical age of $\sim 30000$ years.

According to Morris et al. (1993), the continuum energy distribution of the star can be fitted by a power law of the form $F_{\lambda} \sim \lambda^{-\alpha}$, where $\alpha=2.73$, over the range $1500-9000 \AA$.

A spectrum of the star in the range $3900-4700 \AA$ is shown in Fig. 3 of the paper by Bohannan \& Walborn (1989).

BAT99-134 (Brey 100): Niemela et al. (1991) reported that from their systematic search for high excitation nebulae around $\mathrm{W}-\mathrm{R}$ stars in the Magellanic Clouds, no evidence for nebular HeII $\lambda 4686$ emission in the vicinity of the star could be found.

According to Morris et al. (1993), the continuum energy distribution of the star can be fitted by a power law of the form $F_{\lambda} \sim \lambda^{-\alpha}$, where $\alpha=2.59$, over the range $1500-9000 \AA$.

Dopita et al. (1994) noted that the fine ring nebula, $87^{\prime \prime} \times 107^{\prime \prime}$ in diameter, discovered by Chu \& Lasker (1980), is embedded in a larger shell structure.

Acknowledgements. We gratefully acknowledge the helpful support, comments and criticism of K.A. van der Hucht, G. Meylan, A.F.J. Moffat, D.H. Morgan and N.R. Walborn. We also wish to thank A. Gemmo and O. Hainaut for their assistance in the measurements of the star coordinates. We appreciate the valuable suggestions of the referee, Virpi Niemela, regarding the presentation.

\section{References}

Allen D.A., Glass I.S., 1976, ApJ 210, 666

Andrews A.D., Lindsay E.M., 1964, Ir. Astron. J. 6, 241

Ardeberg A., Brunet J.-P., Maurice E., Prévot L., 1972, A\&AS 6,249

Azzopardi M., Breysacher J., 1979, A\&A 75, 243

Azzopardi M., Breysacher J., 1980, A\&AS 39, 19

Azzopardi M., Breysacher J., 1985, A\&A 149, 213

Barlow M.J., Hummer D.G., 1982, in de Loore C.W.H., Willis A.J. (eds.) Proc. IAU Symp. 99, Wolf-Rayet Stars: Observations, Physics, Evolution. Reidel, Dordrecht, p. 387

Bartzakos P., 1998, PhD Thesis, University of Montréal

Bartzakos P., Moffat A.F.J., Niemela V.S., 1995, in van der Hucht K.A., Williams P.E. (eds.) Proc. IAU Symp. 163, Wolf-Rayet Stars: Binaries Colliding Winds, Evolution. Kluwer, Dordrecht, p. 406

Bastian U., Röser S., Nesterov V.V., Polozhentsev D.D., Potter Kh. I., Wielen R., Yagudin L.I., Yatskiv Ya. S., 1991, A\&AS 87, 159

Bauer T., Weghorn H., Grebel E.K., Bomans D.J., 1996, A\&A 305,135

Beals C.S., 1938, Report of Sub-Committee on the Classification of Wolf-Rayet Stars, IAU Trans. 6, 248

Bohannan B., Epps H.W., 1974, A\&AS 18, 47

Bohannan B., Walborn N.R., 1989, PASP 101, 520

Brandl B., Sams B.J., Bertoldi F., Eckart A., Genzel R., Drapatz S., Hofmann R., Löwe M., Quirrenbach A., 1996, ApJ 466, 254

Breysacher J., 1981, A\&AS 43, 203

Breysacher J., 1986, A\&A 160, 185

Breysacher J., 1988, PhD Thesis, Paris VII University

Buscombe W., Gascoigne S.C.B., de Vaucouleurs G., 1954, Austr. J. Sci. Suppl. 17, No. 3

Campbell B., Hunter D.A., Holtzman J.A., Lauer T.R., Shaya E.J., Code A., Faber S.M., Groth E.J., Light R.M., Lynds R., O’Neil Jr. E.J., Westphal J.A., 1992, AJ 104, 1721

Cannon A.J., 1924, Harvard Obs. Bull., No. 801

Cannon A.J., 1933, Harvard Obs. Bull., No. 891

Chu Y.-H., 1981, ApJ 249, 195

Chu Y.-H., 1983, ApJ 269, 202

Chu Y.-H., Lasker B.M., 1980, PASP 92, 730

Conti P.S., 1982, in de Loore C.W.H., Willis A.J. (eds.) Proc. IAU Symp. 99, Wolf-Rayet Stars: Observations, Physics, Evolution. Reidel, Dordrecht, p. 551

Conti P.S., Garmany C.D., 1983, PASP 95, 411

Conti P.S., Leep E.M., Perry D.N., 1983, ApJ 268, 228

Conti P.S., Massey P., 1989, ApJ 337, 251

Cowley A.P., Crampton D., Hutchings J.B., Thompson I.B., 1984, PASP 96, 968

Cowley A.P., Hutchings J.B., 1978, PASP 90, 636

Crowther P.A., De Marco O., Barlow M.J., 1998, MNRAS 296, 367

Crowther P.A., Dessart L., 1998, MNRAS 296, 622

Crowther P.A., Hillier D.J., Smith L.J., 1995, A\&A 293, 172

Crowther P.A., Smith L.J., 1997, A\&A 320, 500

Crowther P.A., Smith L.J., Willis A.J., 1995, A\&A 304, 269

Davies R.D., Elliott K.H., Meaburn J., 1976, Mem. R. Astr. Soc. 81,89

Dopita M.A., Bell J.F., Chu Y.-H., Lozinskaya T.A., 1994, ApJS 93, 455 
Feast M.W., Thackeray A.D., Wesselink A.J., 1960, MNRAS 121,337

Fehrenbach Ch., Duflot M., Acker A., 1976, A\&AS 24, 379

Feitzinger J.V., Isserstedt J., 1983, A\&AS 51, 505

Fitzpatrick E.L., 1982, ApJ 261, L91

Garnett D.R., Chu Y.-H., 1994, PASP 106, 626

van Genderen A.M., Sterken C., 1996, A\&A 308, 763

Heap S.R., Altner B., Ebbets D., Hubeny I., Hutchings J.B., Kudritzki R.P., Voels S.A., Haser S., Pauldrach A., Puls J., Butler K., 1991, ApJ 377, L29

Heap S.R., Ebbets D., Malumuth E.M, Maran S.P., de Koter A., Hubeny I., 1994, ApJ 435, L39

Henize K.G., 1956, ApJS 2, 315

Heydari-Malayeri M., Grebel E.K., Melnick J., Jorda L., 1993, A\&A 278, 11

Heydari-Malayeri M., Melnick J., 1992, A\&A 258, L13

Heydari-Malayeri M., Melnick J., Van Drom E., 1990, A\&A 236, L21

Hiltner W.A., Schild R.E., 1966, ApJ 143, 770

van der Hucht K.A., Conti P.S., Lundström I., Stenholm B., 1981, Space Sci. Rev. 28, 227

van der Hucht K.A., Hidayat B., Admiranto A.G., Supelli K.R., Doom C., 1988, A\&A 199, 217

Hunter D.A., Shaya E.J., Holtzman J.A., Light R.M., O’Neil Jr. E.J., Lynds R., 1995, ApJ 448, 179

Hyland A.R., Thomas J.A., Robinson G., 1978, AJ 83, 20

Kingsburgh R.L., Barlow M.J., Storey P.J., 1995, A\&A 295, 75

Koesterke L., Hamann W.-R., Schmutz W., Wessolowski U., 1991, A\&A 248, 166

de Koter A., Heap S.R., Hubeny I., 1997, ApJ 477, 792

Lee M.G., 1990, Ph.D. Thesis, University of Washington

Lindsay E.M., 1963, Ir. Astron. J. 6, 127

Lortet M.-C., 1991, in van der Hucht K.A., Hidayat B. (eds.) Proc. IAU Symp. 143, Wolf-Rayet Stars and Interrelations with Other Massive Stars in Galaxies. Kluwer, Dordrecht, p. 513

Lortet M.-C., Testor G., Schild H., 1991, in van der Hucht K.A., Hidayat B. (eds.) Proc. IAU Symp. 143, Wolf-Rayet Stars and Interrelations with Other Massive Stars in Galaxies. Kluwer, Dordrecht, p. 644

Lucke P.B., Hodge P.W., 1970, AJ 75, 171

Malumuth E.M., Heap S.R., 1994, AJ 107, 1054

Massey P., Conti P.S., 1983a, ApJ 264, 126

Massey P., Conti P.S., 1983b, PASP 95, 440

Massey P., Hunter D.A., 1998, ApJ 493, 180

Mathewson D.S., Ford V.L., Dopita M.A., Tuohy I.R., Long K.S., Helfand D.J., 1983, ApJS 51, 345

Mathewson D.S., Ford V.L., Tuohy I.R., Mills B.Y., Turtle A.J., Helfand D.J., 1985, ApJS 58, 197

Melnick J., 1978, A\&AS 34, 383

Melnick J., 1985, A\&A 153, 235

Melnick J., Heydari-Malayeri M., 1991, in van der Hucht K.A., Hidayat B. (eds.) Proc. IAU Symp. 143, Wolf-Rayet Stars and Interrelations with Other Massive Stars in Galaxies. Kluwer, Dordrecht, p. 409

Meylan G., 1993, in Smith G., Brodie J.P. (eds.) $11^{\text {th }}$ Santa Cruz Summer Workshop, The Globular Cluster - Galaxy Connection, San Francisco: Astronomical Society of the Pacific, Vol. 48, p. 588

Moffat A.F.J., 1989, ApJ 347, 373
Moffat A.F.J., 1991, A\&A 244, L9

Moffat A.F.J., 1998 (private communication)

Moffat A.F.J., Niemela V.S., Marraco H.G., 1990, ApJ 348, 232

Moffat A.F.J., Niemela V.S., Phillips M.M., Chu Y.-H., Seggewiss W., 1987, ApJ 312, 612

Moffat A.F.J., Seggewiss W., 1986, ApJ 309, 714

Morgan D.H., 1999, MNRAS (in press)

Morgan D.H., Good A.R., 1985, MNRAS 216, 459

Morgan D.H., Good A.R., 1987, MNRAS 224, 435

Morgan D.H., Good A.R., 1990, MNRAS 243, 459

Morgan D.H., Watson F.G., Parker Q.A., 1992, A\&AS 93, 495

Morris P.W., Brownsberger K.R., Conti P.S., Massey P., Vacca W.D., 1993, ApJ 412, 324

Niemela V.S., 1991, in van der Hucht K.A., Hidayat B. (eds.) Proc. IAU Symp. 143, Wolf-Rayet Stars and Interrelations with Other Massive Stars in Galaxies. Kluwer, Dordrecht, p. 201

Niemela V.S., 1995, in van der Hucht K.A., Williams P.E. (eds.) Proc. IAU Symp. 163, Wolf-Rayet Stars: Binaries, Colliding Winds, Evolution. Kluwer, Dordrecht, p. 223

Niemela V.S., Heathcote S.R., Weller W.G., 1991, in van der Hucht K.A., Hidayat B. (eds.) Proc. IAU Symp. 143, WolfRayet Stars and Interrelations with Other Massive Stars in Galaxies. Kluwer, Dordrecht, p. 425

Niemela V.S., Seggewiss W., Moffat A.F.J., 1995, in van der Hucht K.A., Williams P.E. (eds.) Proc. IAU Symp. 163, Wolf-Rayet Stars: Binaries Colliding Winds, Evolution. Kluwer, Dordrecht, p. 251

Nota A., Leitherer C., Clampin M., Gilmozzi R., 1991, in van der Hucht K.A., Hidayat B. (eds.) Proc. IAU Symp. 143, Wolf-Rayet Stars and Interrelations with Other Massive Stars in Galaxies. Kluwer, Dordrecht, p. 561

Nota A., Pasquali A., Drissen L., Leitherer C., Robert C., Moffat A.F.J., Schmutz W., 1996, ApJS 102, 383

Pakull M.W., 1991, in van der Hucht K.A., Hidayat B. (eds.) Proc. IAU Symp. 143, Wolf-Rayet Stars and Interrelations with Other Massive Stars in Galaxies. Kluwer, Dordrecht, p. 391

Parker J.Wm., 1993, AJ 106, 560

Pasquali A., Langer N., Schmutz W., Leitherer C., Nota A., Hubeny I., Moffat A.F.J., 1997, ApJ 478, 340

Pasquali A., Nota A., Clampin M., 1998, A\&A (in press)

Payne C.H., 1930, Harvard Obs. Bull., No. 878

Phillips M.M., 1982, MNRAS 198, 1053

Rousseau J., Martin N., Prévot L., Rebeirot E., Robin A., Brunet J.-P., 1978, A\&AS 31, 243

Rustamov D.N., Cherepashchuk A.M., 1987, Sov. Astron. Lett. 13,286

Sanduleak N., 1969, Cerro Tololo Inter-American Obs. Contr. No. 89

Schertl D., Hofmann K.-H., Seggewiss W., Weigelt G., 1995, A\&A 302, 327

Schild H., Testor G., 1992, A\&AS 92, 729

Schmutz W., Leitherer C., Hubeny I., Vogel M., Hamann W.-R., Wessolowski U., 1991, ApJ 372, 664

Seggewiss W., Moffat A.F.J., Lamontagne R., 1991, A\&AS 89, 105

Smith L.F., 1968a, MNRAS 138, 109

Smith L.F., 1968b, MNRAS 140, 409 
Smith L.F., 1991, in van der Hucht K.A., Hidayat B. (eds.) Proc. IAU Symp. 143, Wolf-Rayet Stars and Interrelations with Other Massive Stars in Galaxies. Kluwer, Dordrecht, p. 601

Smith L.F., Shara M.M., Moffat A.F.J., 1990a, ApJ 348, 471

Smith L.F., Shara M.M., Moffat A.F.J., 1990b, ApJ 358, 229

Smith L.F., Shara M.M., Moffat A.F.J., 1996, MNRAS 281, 163

Smith L.J., Crowther P.A., Prinja R.K., 1994, A\&A 281, 833

Smith L.J., Nota A., Pasquali A., Leitherer C., Clampin M., Crowther P.A., 1998, ApJ 503, 278

Spoon H.W., de Koter A., Sterken C., Lamers H.J.G.L., Stahl O., 1994, A\&AS 106, 141

Stahl O., Wolf B., Leitherer C., Zickgraf F.-J., Krautter J., de Groot M., 1984, A\&A 140, 459

Testor G., Llebaria A., Debray B., 1988, ESO Messenger, No. 54 , p. 43

Testor G., Niemela V.S., 1998, A\&AS 130, 527

Testor G., Schild H., 1990, A\&A 240, 299

Testor G., Schild H., Lortet M.C., 1993, A\&A 280, 426

Torres A.V., Conti P.S., Massey P., 1986, ApJ 300, 379

Torres-Dodgen A.V., Massey P., 1988, AJ 96, 1076

de Vaucouleurs G., Freeman K.C., 1972, in A. Beer (ed.) Vistas in Astronomy, Vol. 14. Pergamon Press, Oxford \& New York, p. 163
Vanbeveren D., Conti P.S., 1980, A\&A 88, 230

Walborn N.R., 1974, ApJ 189, 269

Walborn N.R., 1977, ApJ 215, 53

Walborn N.R., 1982a, ApJ 254, L15

Walborn N.R., 1982b, ApJ 256, 452

Walborn N.R., 1986, in de Loore C.W.H., Willis A.J., Laskarides P. (eds.) Proc. IAU Symp. 116, Luminous Stars and Associations in Galaxies. Reidel, Dordrecht, p. 185

Walborn N.R., Blades J.C., 1997, ApJS 112, 457

Walborn N.R., Drissen L., Parker J.Wm., Saha A., 1998, in van der Hucht K.A., Koenigsberger G., Eenens P.R.J. (eds.) Proc. IAU Symp. 193, Wolf-Rayet Phenomena in Massive Stars and Starburst Galaxies (in press)

Walborn N.R., MacKenty J.W., Saha A., White R.L., 1995, ApJ 439, L47

Weigelt G., Albrecht R., Barbieri C., et al., 1991, ApJ 378, L21

Weigelt G., Baier G., 1985, A\&A 150, L18

West R.M., 1981, POS Programmes User Manual. ESO Internal Report, Garching

Westerlund B.E., 1961, Uppsala Astron. Obs. Ann. 5, 1

Westerlund B.E., Rodgers A.W., 1959, Observatory 79, 132

Westerlund B.E., Smith L.F., 1964, MNRAS 128, 311 

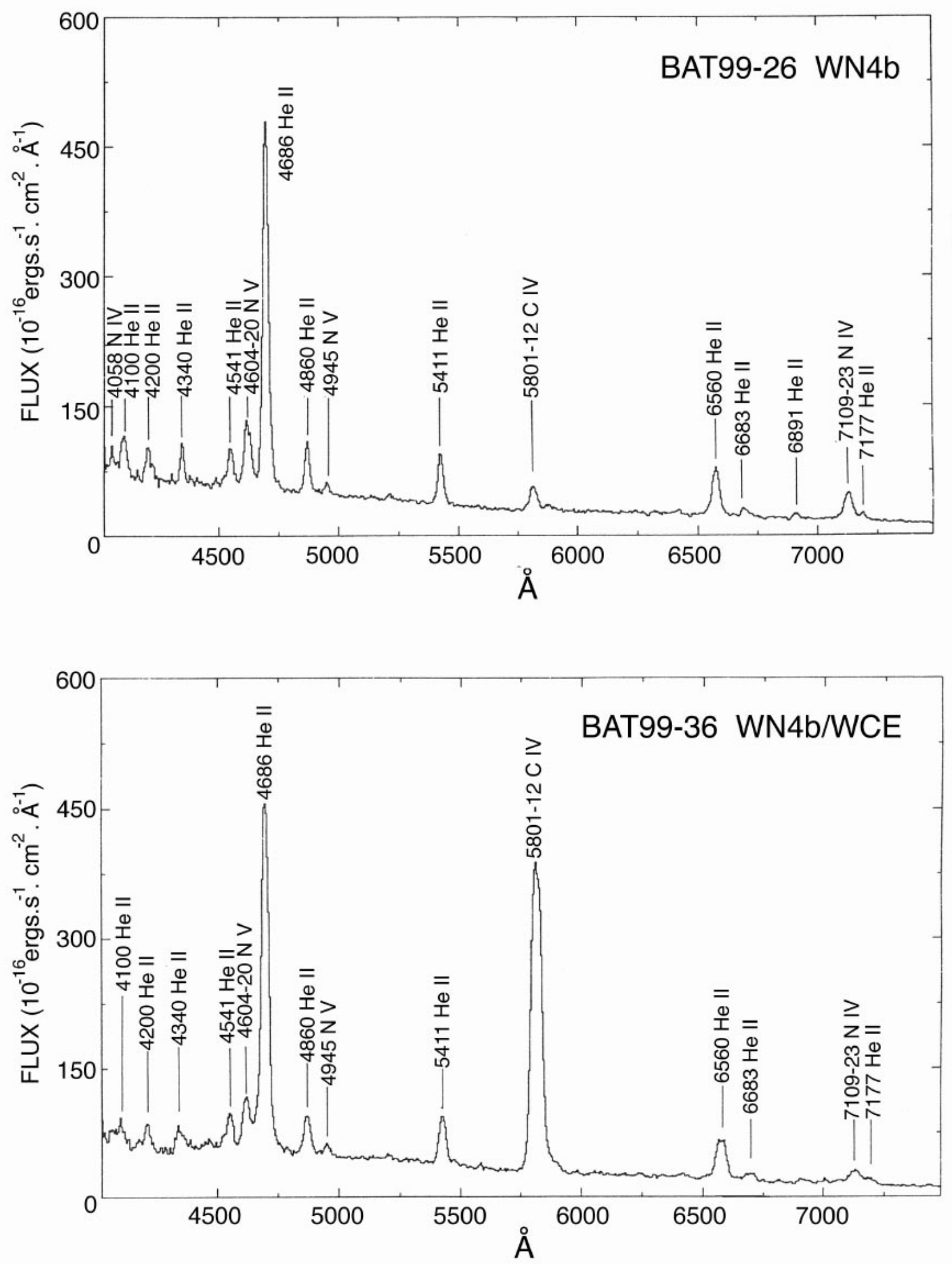

Fig. 1. a) Spectrograms of W - R stars in the LMC: the WN4b star BAT99-26 (Brey 20), and the peculiar WN4b/WCE star BAT99-36 (Brey 29) which shows an unusual strong CIV $\lambda 5801-12$ emission line for a WN-type star 

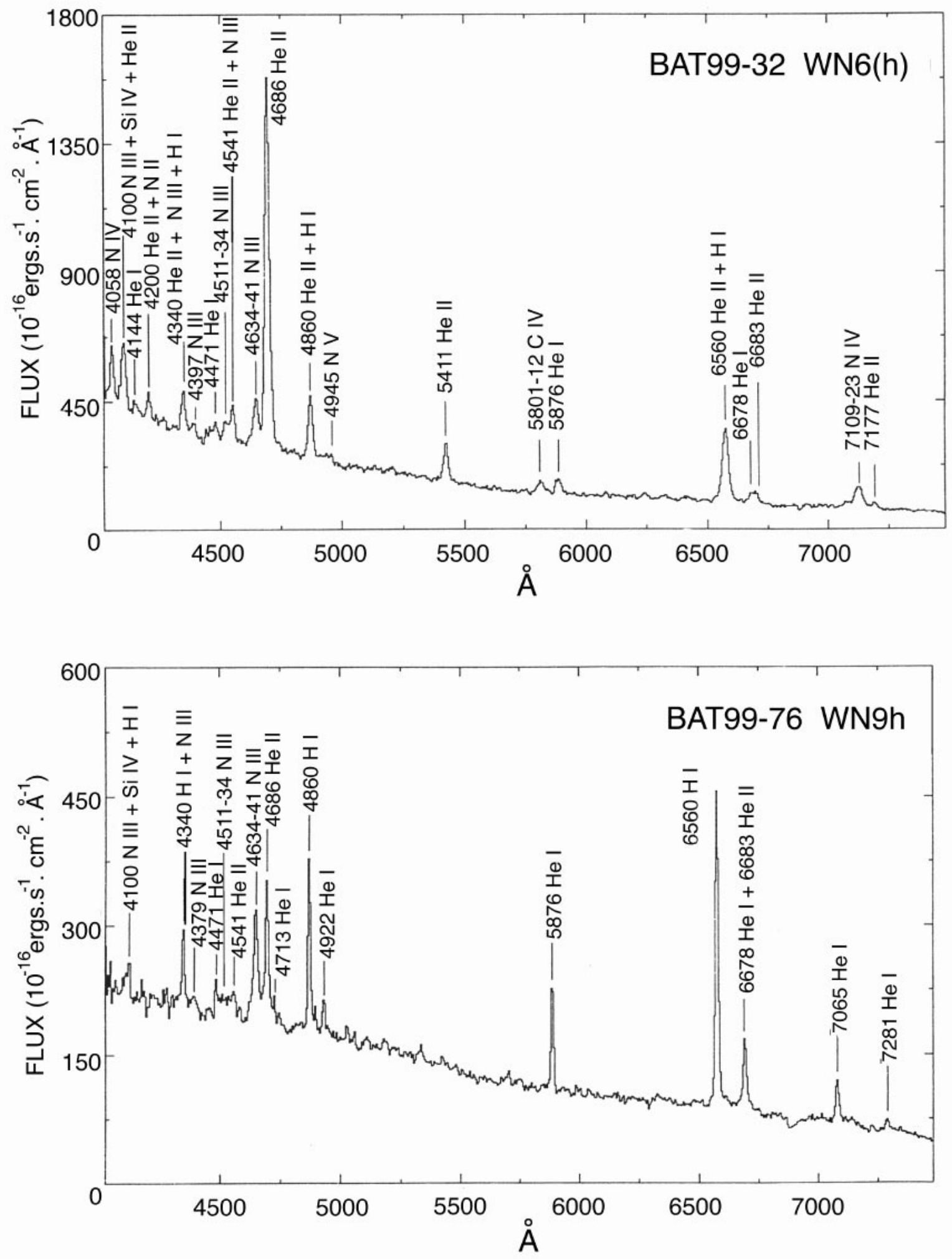

Fig. 1. b) Spectrograms of $\mathrm{W}-\mathrm{R}$ stars in the LMC: the WN6(h) star BAT99-32 (Brey 26), and the WN9h star BAT99-76 (Brey 64) 

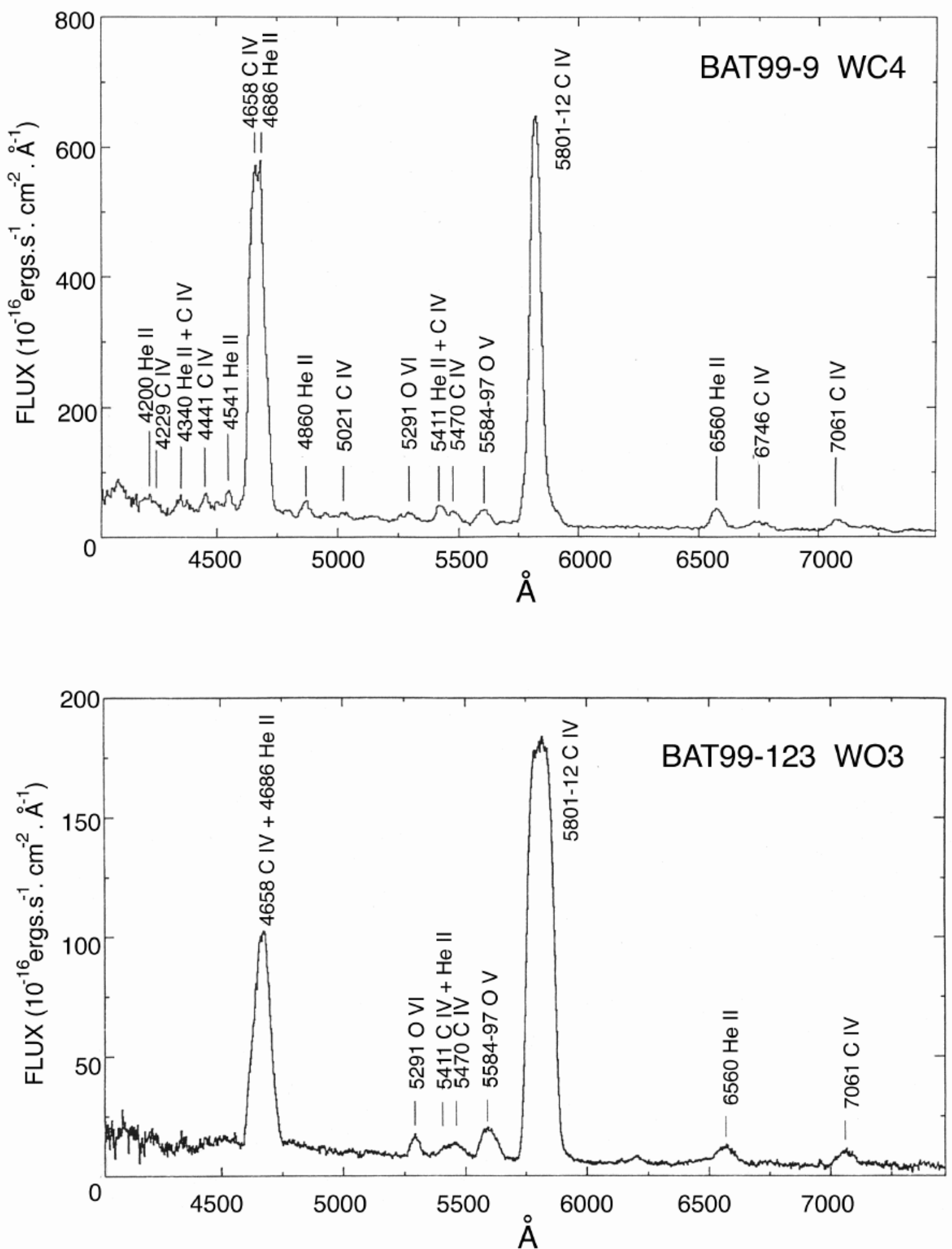

Fig. 1. c) Spectrograms of W - R stars in the LMC: the WC4 star BAT99-9 (Brey 7), and the WO3 star BAT99-123 (Brey 93) 
Table 2. Wolf-Rayet stars in the Large Magellanic Cloud

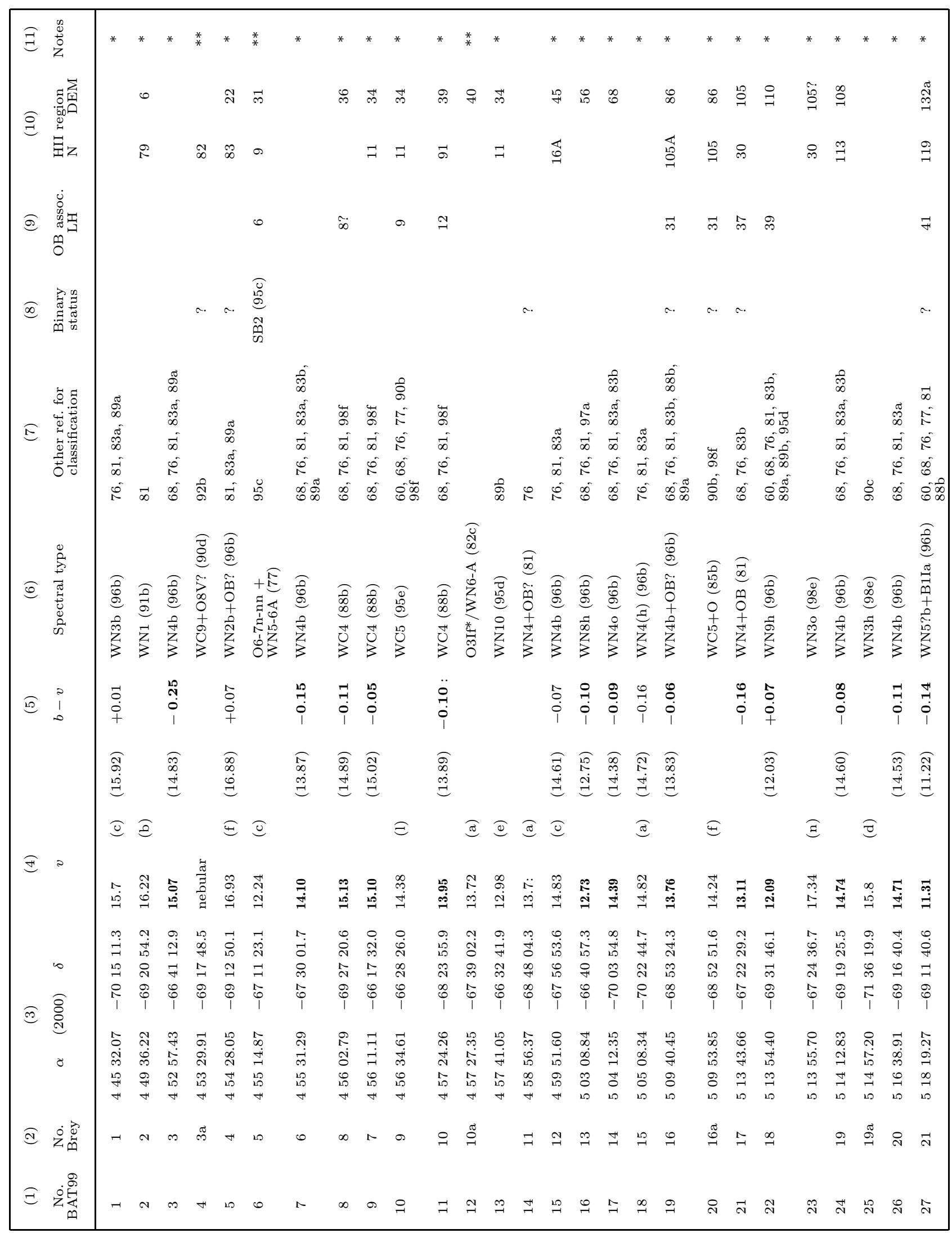


Table 2. continued

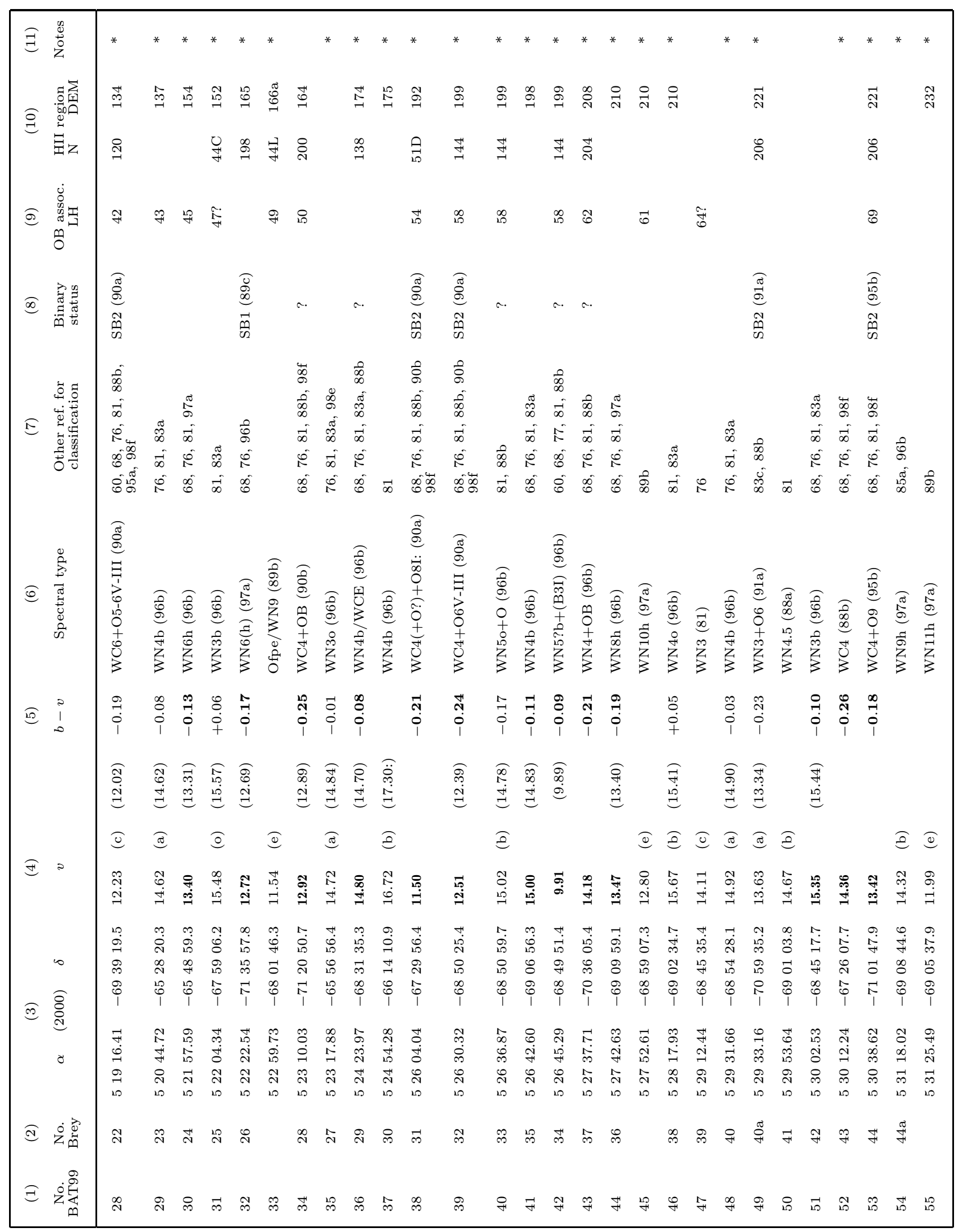


Table 2. continued

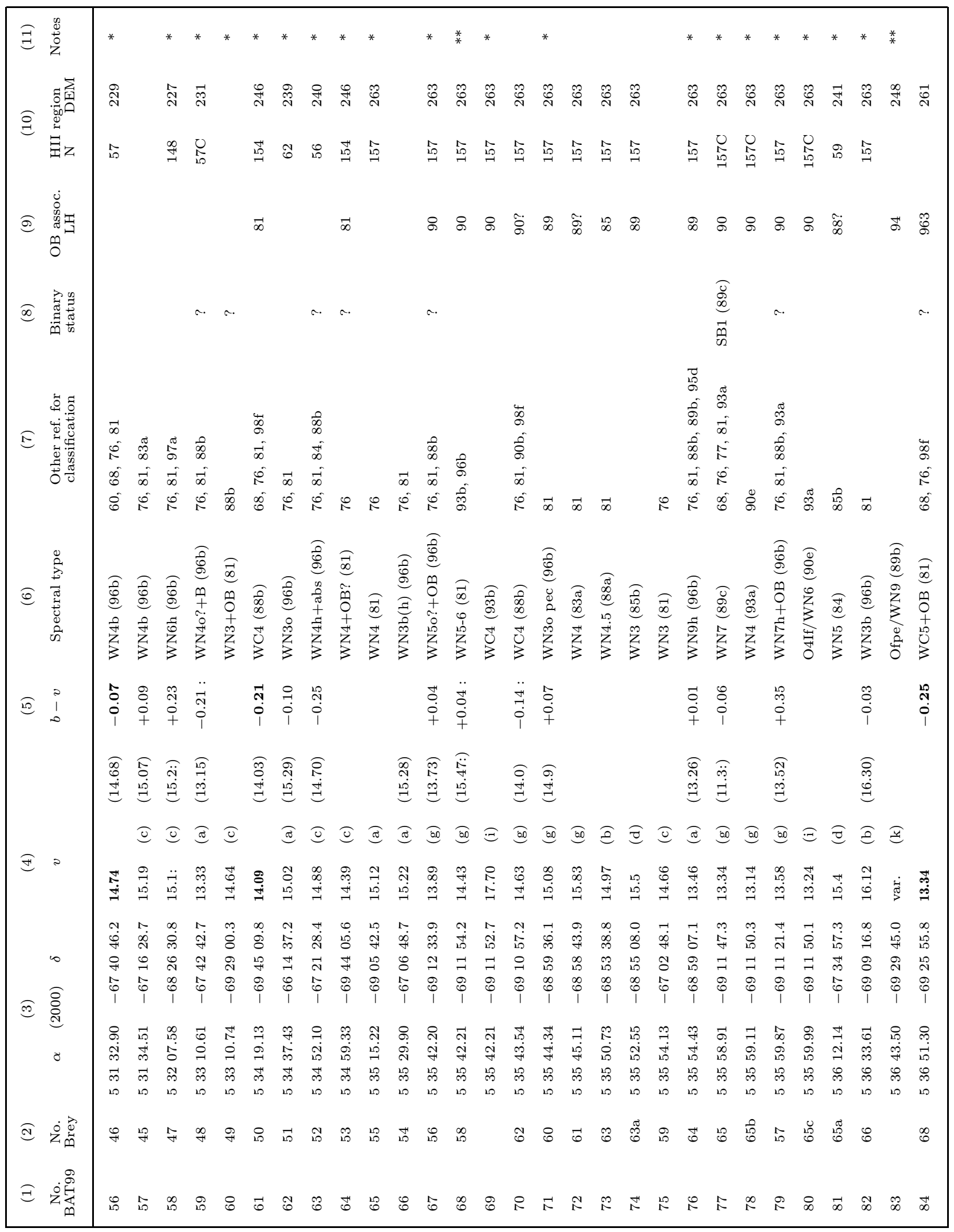


Table 2. continued

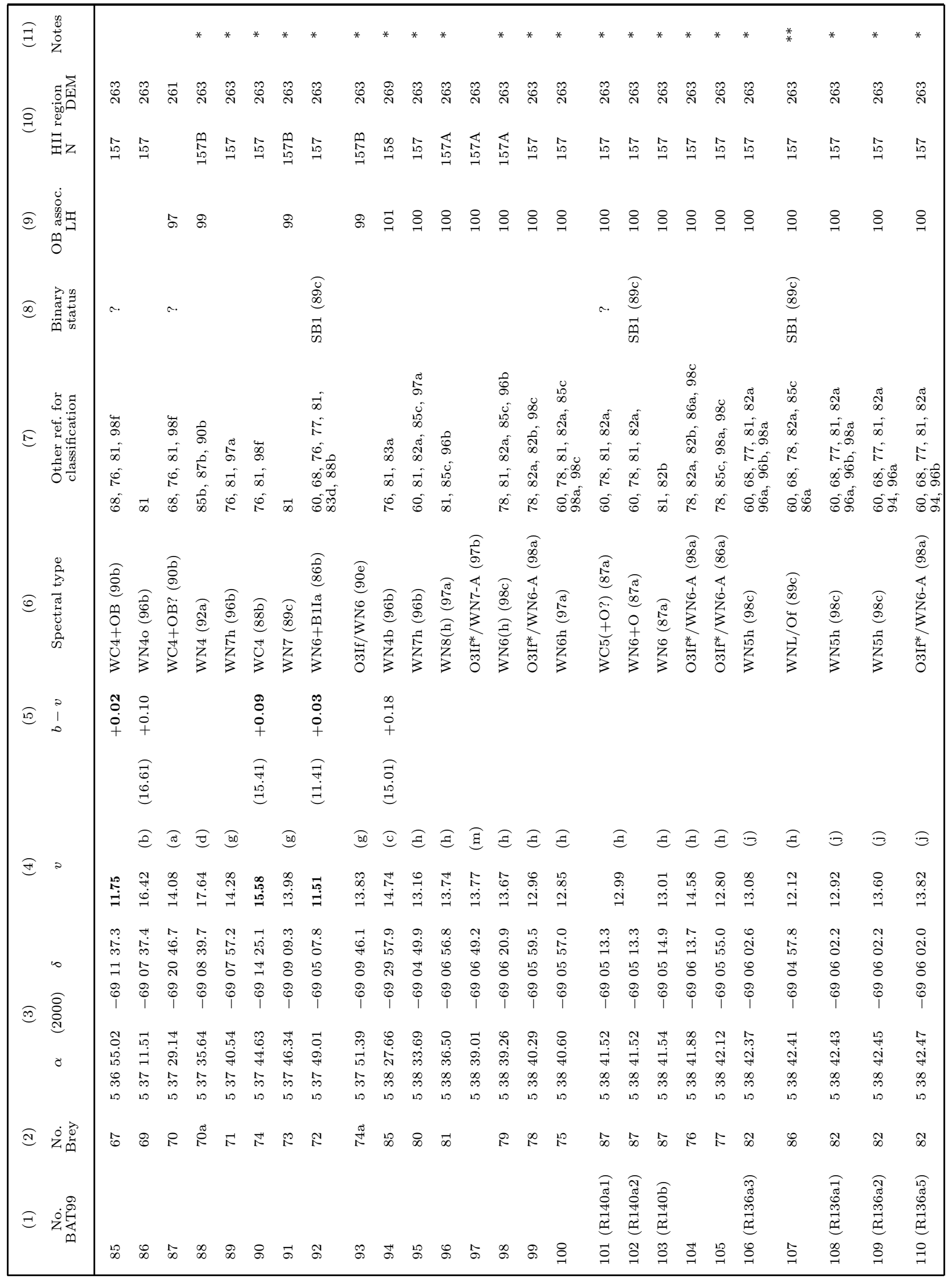


Table 2. continued

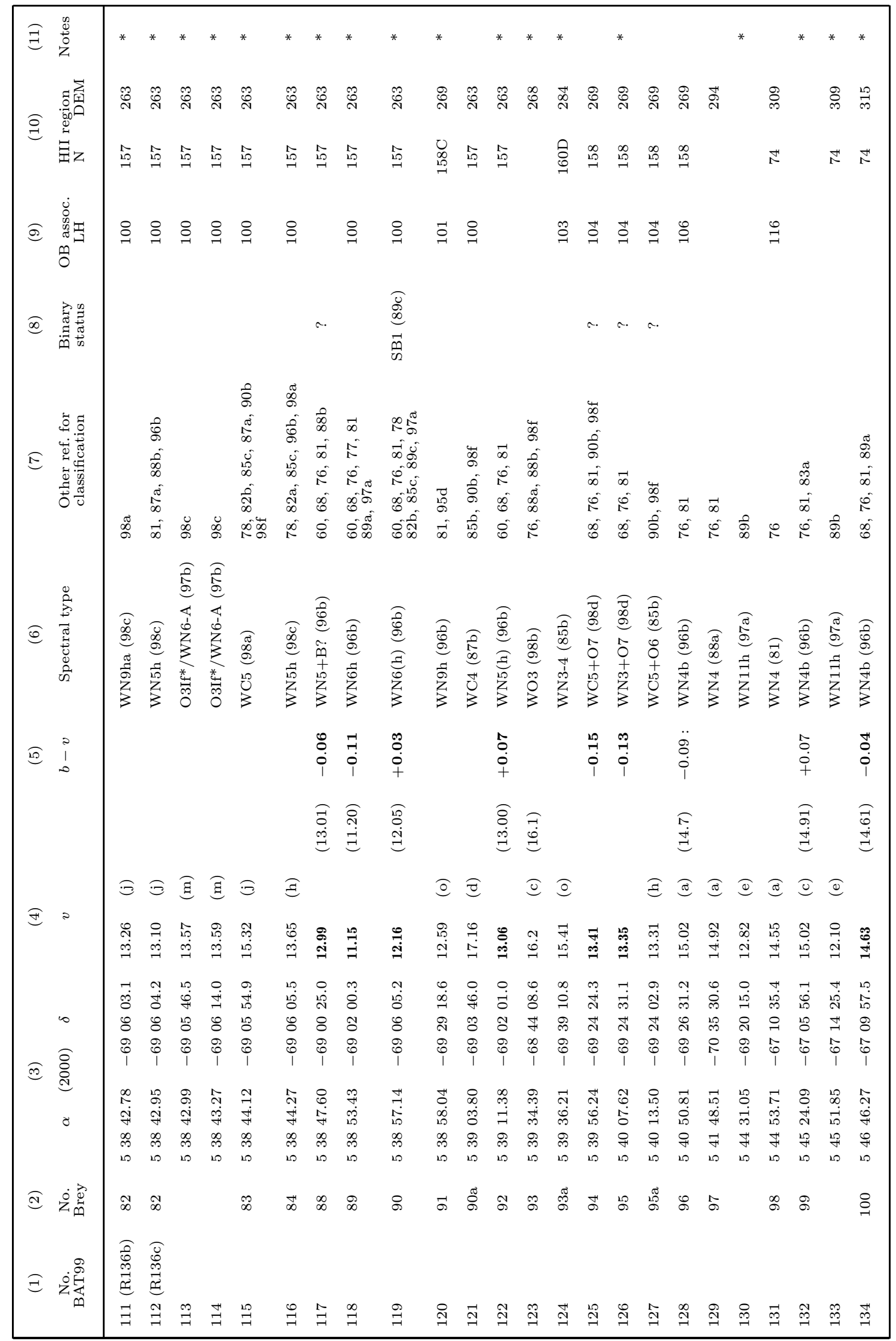


Table 3. Cross-identifications for Wolf-Rayet stars in the Large Magellanic Cloud

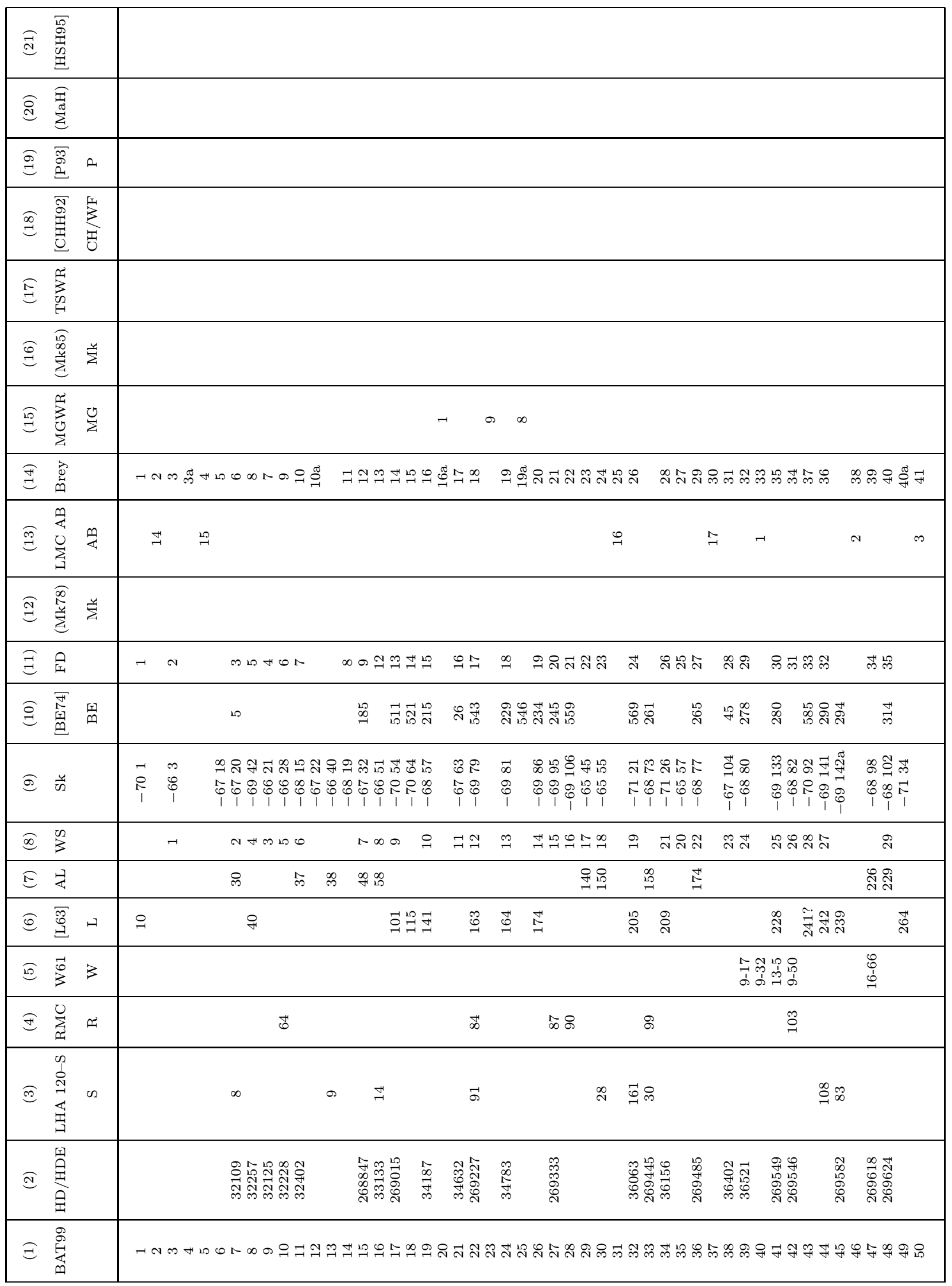


Table 3. continued

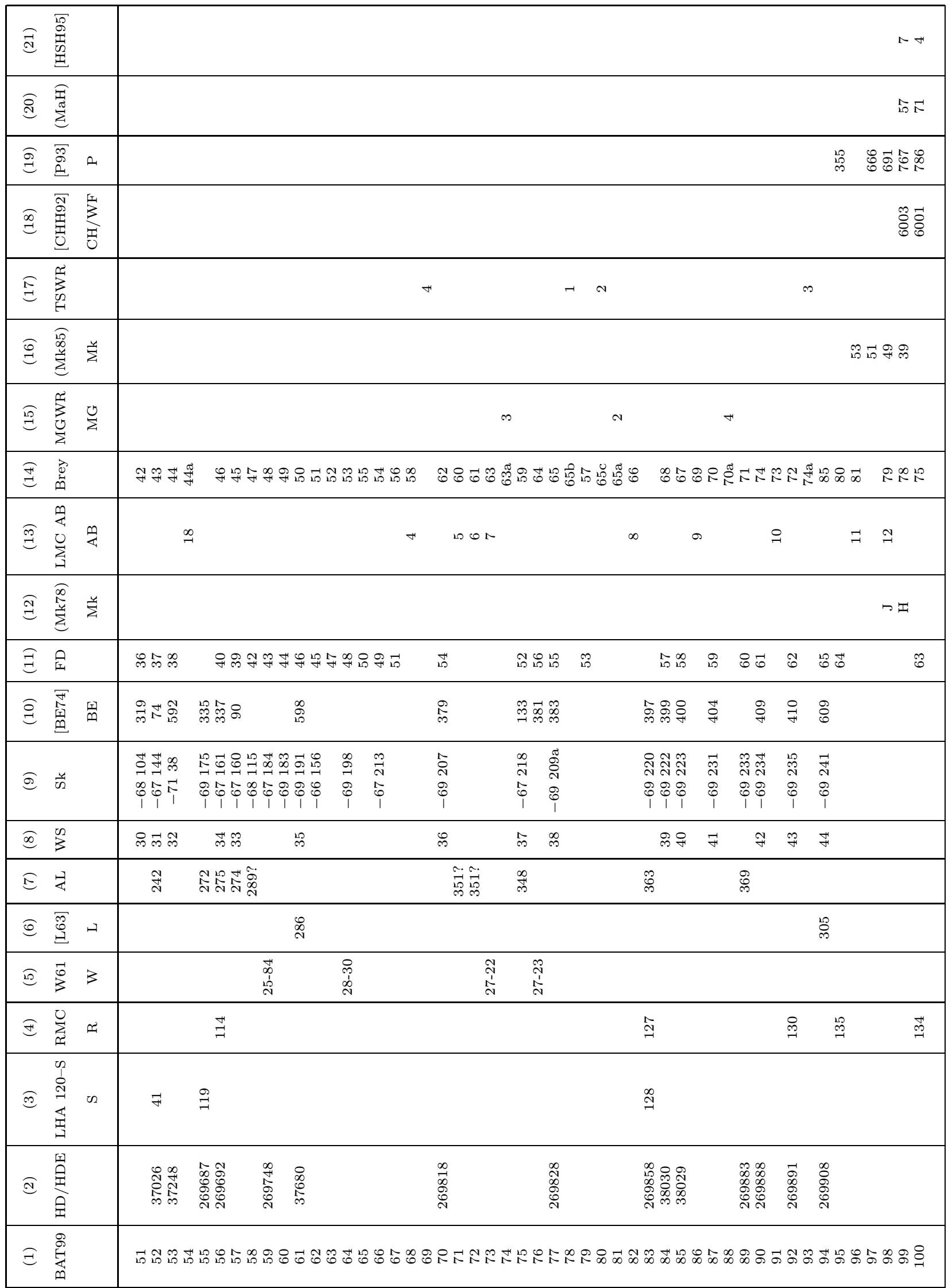


Table 3. continued

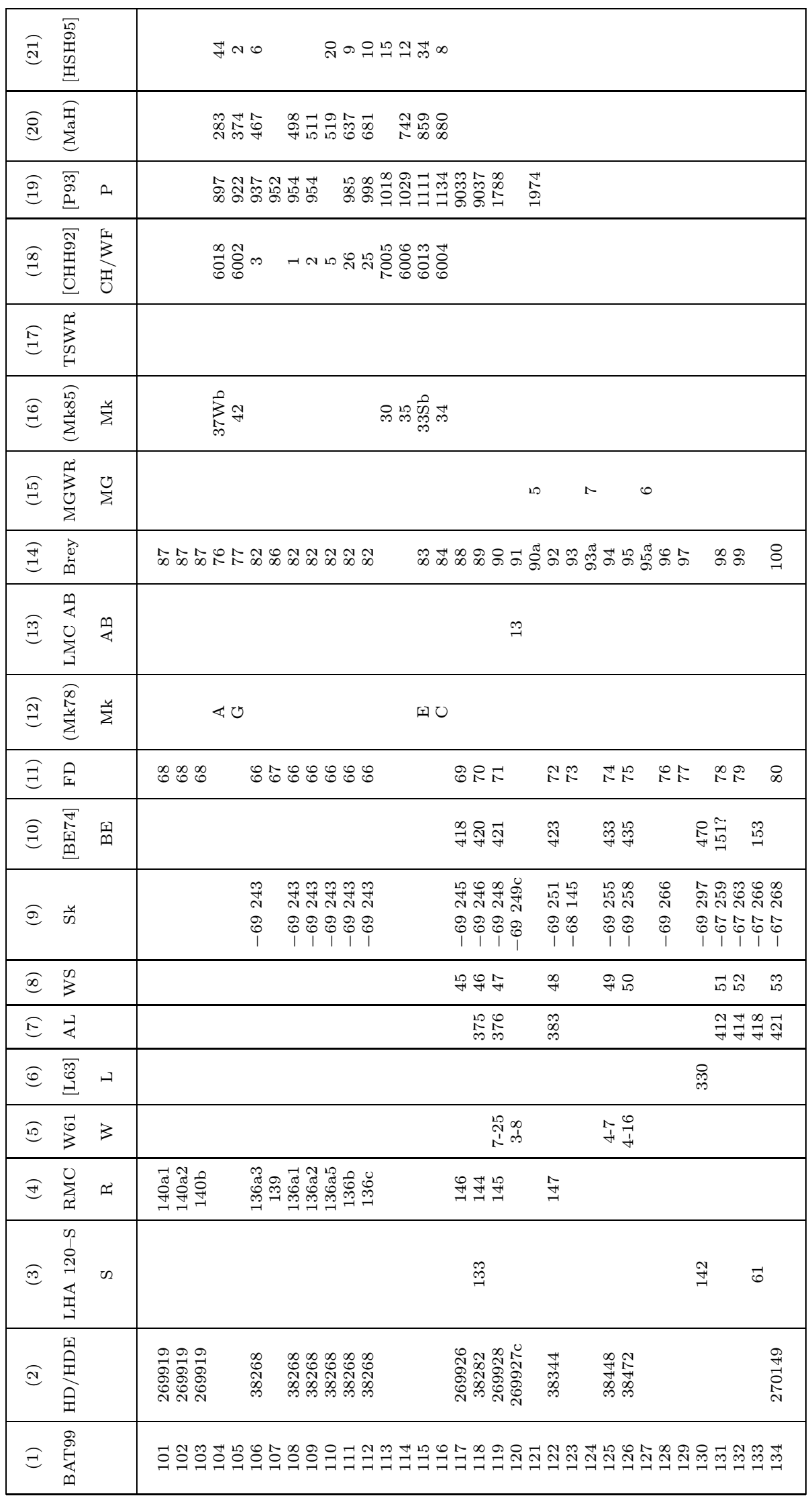

